Pacific

Journal of

Mathematics

LOCAL CUT POINTS AND METRIC MEASURE SPACES WITH RICCI CURVATURE BOUNDED BELOW

MASAYOSHI WATANABE

Volume $233 \quad$ No. 1

November 2007 


\title{
LOCAL CUT POINTS AND METRIC MEASURE SPACES WITH RICCI CURVATURE BOUNDED BELOW
}

\author{
MASAYOSHI WATANABE
}

\begin{abstract}
A local cut point is a point that disconnects its sufficiently small neighborhood. We show that there exists an upper bound for the degree of a local cut point in a metric measure space satisfying the generalized Bishop-Gromov inequality. As a corollary, we obtain an upper bound for the number of ends of such a space. We also obtain some obstruction conditions for the existence of a local cut point in a metric measure space satisfying the Bishop-Gromov inequality or the Poincaré inequality. For example, the measured GromovHausdorff limits of Riemannian manifolds with a lower Ricci curvature bound satisfy these two inequalities.
\end{abstract}

\section{Introduction}

A point $x$ in a metric space is called a local cut point if $U \backslash\{x\}$ is disconnected for some connected neighborhood $U$ of $x$. For $n \geq 2$, there exists no local cut point in any $n$-dimensional Alexandrov space with curvature bounded from below. Every Gromov-Hausdorff limit of a sequence of Riemannian manifolds with a uniform lower bound on sectional curvature is such an Alexandrov space. It is therefore natural to ask whether there exists a local cut point in the limit of manifolds with a uniform lower bound on Ricci curvature unless the limit is one-dimensional. We conjecture that the limit has no local cut point.

In this paper, we consider metric measure spaces with (suitably defined) Ricci curvature bounded below. Let $\operatorname{deg}(x)$ denote the degree of a point $x$ or the supremum of the number of connected components of $U \backslash\{x\}$ for all connected neighborhoods $U$ of $x$. We give an upper bound for the degree of a local cut point. As a consequence, we obtain an upper bound for the number of ends (see Section 4.2 for the definition of an end). We also obtain some obstruction conditions for the existence of a local cut point.

MSC2000: 53C21, 53C23, 31C15.

Keywords: local cut points, Ricci curvature, metric measure spaces, ends, Gromov-Hausdorff convergence, Bishop-Gromov inequality, Poincaré inequality.

This work was partially supported by Research Fellowships of the Japan Society for the Promotion of Science for Young Scientists. 
Cheeger and Colding [1996; 1997; 2000a; 2000b] and Menguy [2000a; 2000b; 2001] studied the limits of manifolds with Ricci curvature bounded from below. They constructed examples of limit spaces showing that the local structure is more complicated than that of Alexandrov spaces. Recently, Lott and Villani $[\geq 2007$; 2007], Sturm [2006a; 2006b], and Ohta [2007] independently generalized to metric measure spaces the notion of Ricci curvature bounded below, introducing the condition that a space has $N$-Ricci curvature $\geq K$, the curvature-dimension condition $\operatorname{CD}(K, N)$, and the measure contraction property $\operatorname{MCP}(K, N)$. For each $N$-dimensional Riemannian manifold $M$, each of these conditions is equivalent to the Ricci curvature of $M$ being bounded below by $K$. Moreover, the conditions are preserved under measured Gromov-Hausdorff limits.

We note that the measured Gromov-Hausdorff limits of manifolds with Ricci curvature bounded from below satisfy the generalized Bishop-Gromov inequality and a Poincaré inequality of type $(1,1)$, as explained below.

Let $(X, d, \mu)$ be a complete, locally compact length space equipped with a Borel measure. We denote by $B_{r}(x)$ the open ball of radius $r$ and centered at $x \in X$. Let $k \in \mathbb{R}, n \in \mathbb{N}, C \geq 1$. Let $0<r \leq R$ and $x \in X$. If $C=1$, then (1-1) is the usual Bishop-Gromov inequality with respect to lower bound $(n-1) k$ of Ricci curvature and upper bound $n$ of dimension:

$$
\frac{\mu\left(B_{R}(x)\right)}{\mu\left(B_{r}(x)\right)} \leq C \frac{V_{k, n}(R)}{V_{k, n}(r)}
$$

where $V_{k, n}(r)$ is the volume of a ball of radius $r$ in the $n$-dimensional, complete, simply connected space of constant curvature $k$.

We consider a stronger inequality, which is the directionally restricted version of (1-1). See [Gromov 1999, Section 5. I+] and [Cheeger and Colding 1997, Appendix 2, (A.2.2.)]. We call the inequality the generalized Bishop-Gromov inequality with constant $C$ or, for short, $\mathrm{BG}(k, n)$ with $C$. This inequality naturally extends to the case $n \in \mathbb{R}$ with $n \geq 1$. See Definition 3.1 for the precise definition and [Kuwae and Shioya 2001] for slightly different definitions. This inequality is preserved under the measured Gromov-Hausdorff limits; see [Gromov 1999, Section 5. $\mathrm{I}_{+}$] and [Cheeger and Colding 1997, Theorem 1.6, Theorem 1.10]. The measure contraction property $\operatorname{MCP}((n-1) k, n)$ implies $\mathrm{BG}(k, n)$ with $C=1$. For example, these metric measure spaces satisfy $\operatorname{MCP}((n-1) k, n)$ :

- $n$-dimensional Riemannian manifolds whose Ricci curvature is bounded below by $(n-1) k$ and that are equipped with a Riemannian measure;

- $n$-dimensional Alexandrov spaces whose curvature is bounded below by $k$ and that are equipped with the $n$-dimensional Hausdorff measure; 
- "nonbranching" metric measure spaces satisfying the curvature-dimension condition $\mathrm{CD}((n-1) k, n)$ (see Definition 3.3 for "nonbranching").

The non-Euclidean, finite-dimensional, normed linear spaces equipped with the Lebesgue measure satisfy $\operatorname{MCP}(0, n)$. These spaces, however, cannot arise as a Gromov-Hausdorff limit of any Riemannian manifolds with a uniform lower bound on Ricci curvature (see Proposition 3.2).

Applying the method of the proof of Theorem 5.1 in [Cheeger and Colding 2000a], we have

Theorem 1.1. Let $(X, d, \mu)$ be a metric measure space satisfying the generalized Bishop-Gromov inequality $\mathrm{BG}(k, n)$ with constant $C$ for some $k \in \mathbb{R}, n \geq 1$, and $C \geq 1$. Assume that there exists a local cut point $x$ in $X$. Then $\operatorname{deg}(x) \leq C^{2}+1$.

The Cheeger-Gromoll splitting theorem [1971/72] states that if a Riemannian manifold $M$ of nonnegative Ricci curvature contains a straight line, then $M$ is isometric to $\mathbb{R} \times N$ for some manifold $N$. Cheeger and Colding [1996] extended this to limit spaces of nonnegative Ricci curvature in a generalized sense; see our Theorem 2.1. For the limits of manifolds with Ricci curvature bounded from below $(C=1)$, Theorem $1.1(\operatorname{deg}(x)=2)$ is also proved by using the Cheeger-Colding splitting theorem; see Proposition 4.3 here.

By the splitting theorem, a space of nonnegative Ricci curvature has at most two ends. The splitting theorem for metric measure spaces satisfying $\mathrm{BG}(0, n)$ with $C$ does not necessarily hold. As a corollary of Theorem 1.1, we have

Corollary 1.2. Let $(X, d, \mu)$ be a metric measure space satisfying $\mathrm{BG}(0, n)$ with $C$ for some $n \geq 1$ and $C \geq 1$. Then the number of ends of $X$ is at most $C^{2}+1$.

We investigate the geometric structure of the neighborhood of a local cut point (Theorem 4.14). As a result, the "three-pronged" space (see Figure 4) does not satisfy $\mathrm{BG}(k, n)$ with $C$. We also study the structure of the accumulation of local cut points and find that the convergent sequence of certain local cut points "stands in a line" (Corollary 4.20).

We now assume that a measure $\mu$ satisfies $0<\mu\left(B_{r}(x)\right)<+\infty$ for all $x \in X$ and all $0<r<+\infty$. Let $1 \leq p<\infty$. We say that a metric measure space $(X, d, \mu)$ satisfies a Poincaré inequality of type $(1, p)$ if, for all $R>0$, there exists a constant $C_{P}=C_{P}(p, R)>0$ depending only on $p$ and $R$ such that

$$
f_{B_{r}(x)}\left|u-u_{B_{r}(x)}\right| d \mu \leq C_{P} r\left(f_{B_{r}(x)} g^{p} d \mu\right)^{1 / p}
$$

holds for all $x \in X$, the range $0<r \leq R$, all measurable functions $u$, and all "upper gradients" $g$ of $u$ (see Section 4), where $u_{B}:=f_{B} u d \mu:=\mu(B)^{-1} \int_{B} u d \mu$.

It follows from Hölder's inequality that a metric measure space satisfying a Poincaré inequality of type $(1, p)$ also satisfies one of type $(1, q)$ for all $q \geq p$. For 
instance, there are many examples of metric measure spaces satisfying a Poincaré inequality of type $(1,1)$ :

- $n$-dimensional Riemannian manifolds with Ricci curvature bounded below by $(n-1) k$ equipped with Riemannian measure [Buser 1982]. The constant $C_{P}$ in (1-2) depends on $k, n$, and $R$. If $k=0$, then $C_{P}$ depends only on $n$. See [Saloff-Coste 2002, Theorem 5.6.5].

- The measured Gromov-Hausdorff limits of manifolds with Ricci curvature bounded from below [Cheeger and Colding 2000b, Theorem 2.15].

- $n$-dimensional Alexandrov spaces equipped with the $n$-dimensional Hausdorff measure. See [Kuwae et al. 2001].

- Nonbranching metric measure spaces satisfying $\operatorname{CD}(K, N)$. See [von Renesse 2007, Section 3.2], and combine with [Sturm 2006b, Lemma 4.1].

- Metric measure spaces with a doubling measure and the "democratic condition” DM. See [Lott and Villani 2007, Theorem 2.5].

The space $\left\{\left(x_{1}, x_{2}, \ldots, x_{n}\right) \in \mathbb{R}^{n} \mid x_{1}^{2}+x_{2}^{2}+\cdots+x_{n-1}^{2} \leq x_{n}^{2}\right\}$ equipped with the Euclidean distance and the $n$-dimensional Lebesgue measure satisfies a Poincaré inequality of type $(1, p)$ for all $p>n$ [Hajłasz and Koskela 2000, Example 4.2].

We can obtain an obstruction condition for the existence of a local cut point:

Theorem 1.3. Let $(X, d, \mu)$ be a metric measure space satisfying a Poincaré inequality of type $(1, p)$ for some $1 \leq p<\infty$. Assume that

$$
\liminf _{r \rightarrow 0} \frac{\mu\left(B_{r}(x)\right)}{r^{p}}=0
$$

for a point $x \in X$. Then $x$ is not a local cut point.

Although the measured Gromov-Hausdorff limits of manifolds with Ricci curvature bounded from below satisfy a Poincaré inequality of type $(1,1)$, they do not necessarily satisfy the assumption (1-3) for $p=1$. See [Cheeger and Colding 1997, Proposition 1.22]. Theorem 1.3 is one of the geometric consequences of the Poincaré inequality. See [Hajłasz and Koskela 2000, Proposition 4.5] and [Heinonen and Koskela 1998, Theorems 3.13 and 6.15] for related results.

This paper is organized as follows: In Section 2, we recall the definitions of the Hausdorff dimension, length spaces, and the (pointed, measured) GromovHausdorff convergence. In Section 3, we precisely define $\mathrm{BG}(k, n)$ with $C$ and give its basic properties. We prove Theorem 1.1 and Corollary 1.2 in Section 4. We also investigate the geometric structure of the neighborhood of local cut points and also study the structure of the accumulation of local cut points. We prove Theorem 1.3 in Section 5. 


\section{Preliminaries}

We first recall the definition of the Hausdorff dimension. We then define length spaces and the (pointed, measured) Gromov-Hausdorff convergence. Let $(X, d)$ be a metric space.

2.1. Hausdorff dimension. We refer to [Ambrosio and Tilli 2004, Chapter 2] for details. Let $A \subset X$. For $0 \leq s<\infty$ and $0<\delta \leq \infty$, we define

$$
\mathscr{H}_{\delta}^{s}(A)=\omega_{s} \inf \left\{\sum_{i}\left(\frac{\operatorname{diam}\left(U_{i}\right)}{2}\right)^{s} \mid A \subset \bigcup_{i} U_{i}, \operatorname{diam}\left(U_{i}\right) \leq \delta\right\},
$$

where $\omega_{s}:=\pi^{s / 2} / \Gamma(s / 2+1)$ and $\Gamma$ is the gamma function $\Gamma(s)=\int_{0}^{\infty} e^{-x} x^{s-1} d x$. If $s$ is a positive integer, then $\omega_{s}$ equals the volume of a unit ball in $\mathbb{R}^{s}$. Note that $\mathscr{H}_{\delta}^{s}(A)$ is nonincreasing in $\delta$. We define the s-dimensional Hausdorff measure of $A$ by

$$
\mathscr{H}^{s}(A)=\lim _{\delta \rightarrow 0} \mathscr{H}_{\delta}^{s}(A)=\sup _{\delta>0} \mathscr{H}_{\delta}^{s}(A) .
$$

Then $\mathscr{H}^{s}$ is a Borel regular measure on $X$. The Hausdorff dimension of $A$ is

$$
\operatorname{dim}_{\mathrm{H}}(A)=\inf \left\{0 \leq s<\infty \mid \mathscr{H}^{s}(A)=0\right\}=\sup \left\{0 \leq s<\infty \mid \mathscr{H}^{s}(A)=+\infty\right\} .
$$

2.2. Length spaces. We define the length of a continuous path $\gamma:[0, l] \rightarrow X$ by

$$
L(\gamma)=\sup _{0=t_{0}<t_{1}<\cdots<t_{k}=l} \sum_{i=1}^{k} d\left(\gamma\left(t_{i-1}\right), \gamma\left(t_{i}\right)\right),
$$

where the supremum is taken over all partitions of $[0, l]$. By the triangle inequality, we have $L(\gamma) \geq d(\gamma(0), \gamma(l))$. We say that a path $\gamma:[0, l] \rightarrow X$ is a geodesic if it is locally minimizing and is proportional to arclength, that is, for each $s \in[0, l]$ there exists $\epsilon=\epsilon(s)>0$ such that

$$
d\left(\gamma(t), \gamma\left(t^{\prime}\right)\right)=\left|t-t^{\prime}\right|
$$

holds for all $t, t^{\prime} \in(s-\epsilon, s+\epsilon) \cap[0, l]$. Furthermore, we say that a path $\gamma$ : $(-\infty, \infty) \rightarrow X$ is a straight line if (2-4) holds for all $t, t^{\prime} \in(-\infty, \infty)$. In this paper, we assume that every path is proportional to arclength.

We say that $X$ is a length space if $d(x, y)=\inf _{\gamma} L(\gamma)$ for all $x, y \in X$, where the infimum is taken over all paths joining $x$ and $y$. A metric space $X$ is a length space if and only if, for all $x, y \in X$ and all $\epsilon>0$, there exists a point $z \in X$ such that $\max \{d(x, z), d(z, y)\} \leq d(x, y) / 2+\epsilon$. If $X$ is a complete, locally compact length space, then all two points in $X$ are joined by a minimal geodesic.

For more, see [Burago et al. 2001; Gromov 1999] and their references. 
2.3. Gromov-Hausdorff convergence. Let us recall that the Hausdorff distance between two closed bounded subsets $A$ and $B$ in a metric space $X$ is defined by

$$
d_{\mathrm{H}}(A, B)=\inf \left\{\epsilon>0 \mid A \subset U_{\epsilon}(B), B \subset U_{\epsilon}(A)\right\},
$$

where $U_{\epsilon}(A)$ is the $\epsilon$-neighborhood of $A$. Let $\mathscr{C}$ denote the set of isometry classes of all compact metric spaces. For $X, Y \in \mathscr{C}$, the Gromov-Hausdorff distance $d_{\mathrm{GH}}(X, Y)$ between $X$ and $Y$ is defined as the infimum of $d_{\mathrm{H}}(f(X), g(Y))$ over all metric spaces $Z$ and all isometric embeddings $f: X \rightarrow Z$ and $g: Y \rightarrow Z$. Then $d_{\mathrm{GH}}$ defines a metric on $\mathscr{C}$. Let $\left(X, d_{X}\right)$ and $\left(Y, d_{Y}\right)$ be metric spaces. For $\epsilon>0$, we say that a map $\varphi: X \rightarrow Y$ is an $\epsilon$-approximation if

(i) $\left|d_{X}(x, y)-d_{Y}(\varphi(x), \varphi(y))\right|<\epsilon$ holds for all $x, y \in X$, and

(ii) the $\epsilon$-neighborhood of $\varphi(X)$ coincides with $Y$.

Let $X_{i}, X \in \mathscr{C}$ for $i=1,2, \ldots$ The sequence $\left\{X_{i}\right\}$ Gromov-Hausdorff converges to $X$, that is, $d_{\mathrm{GH}}\left(X_{i}, X\right) \rightarrow 0$ as $i \rightarrow \infty$, if and only if there exist $\epsilon_{i}$-approximations from $X_{i}$ to $X$ (or from $X$ to $X_{i}$ ) for some $\epsilon_{i} \rightarrow 0$.

Let $\left(X_{i}, x_{i}\right)$ for $i=1,2, \ldots$ and $(X, x)$ be pointed metric spaces. We say that $\left\{\left(X_{i}, x_{i}\right)\right\}$ pointed Gromov-Hausdorff converges to $(X, x)$ if, for each $R>0$, there exist $R_{i} \searrow R, \epsilon_{i} \searrow 0$, and $\epsilon_{i}$-approximations $\varphi_{i}: B_{R_{i}}\left(x_{i}\right) \rightarrow B_{R}(x)$ with $\varphi_{i}\left(x_{i}\right)=x$. A pointed Gromov-Hausdorff limit of $\left\{\left(X, r_{i}^{-1} d, x\right)\right\}$ as $r_{i} \rightarrow 0$ is called a tangent cone at $x$. The Gromov-Hausdorff limit of a sequence of length spaces is also a length space. See [Burago et al. 2001; Gromov 1999] for details.

Let $\left(M_{i}, p_{i}\right)$ for $i=1,2, \ldots$ be $n$-dimensional, complete pointed Riemannian manifolds with Ricci curvature $\operatorname{Ric}_{M_{i}} \geq-(n-1)$. Gromov's compactness theorem states that $\left\{\left(M_{i}, p_{i}\right)\right\}$ pointed Gromov-Hausdorff subconverges to some pointed metric space $(X, x)$. We then have $\operatorname{dim}_{\mathrm{H}}(X) \leq n$ (see Proposition 3.8). The sequence $\left\{M_{i}\right\}$ is said to collapse to $X$ if $\operatorname{dim}_{H}(X)<n$. In fact, if $\left\{M_{i}\right\}$ collapses, then $\operatorname{dim}_{\mathrm{H}}(X) \leq n-1$ [Cheeger and Colding 1997, Theorem 3.1]. Fukaya conjectured [1987, Conjecture 3.13] that the Hausdorff dimension of the limit $X$ is an integer. By Gromov's compactness theorem, there exists a tangent cone at every $x$ in the limit $X$. A tangent cone at each point is not necessarily unique even in the noncollapsed case [Cheeger and Colding 1997, Example 8.41]. It does not always have a metric cone structure in the collapsed case [Cheeger and Colding 1997, Example 8.95]. The splitting theorem for the limit holds, even in the collapsed case:

Theorem 2.1 [Cheeger and Colding 1996]. Let $(X, x)$ be a pointed GromovHausdorff limit of complete Riemannian manifolds $M_{i}$ with $\operatorname{Ric}_{M_{i}} \geq-\delta_{i}$, where $\delta_{i} \rightarrow 0$. If $X$ contains a straight line, then $X$ is isometric to $\mathbb{R} \times Y$ for some length space $Y$. 
2.4. Measured Gromov-Hausdorff convergence. Fukaya [1987] introduced the concept of the measured Gromov-Hausdorff convergence. Let $\mathscr{b} M$ denote the set of all compact metric spaces $X$ equipped with a Borel measure $\mu$ such that $\mu(X) \leq 1$. A directed system $\left\{\left(X_{\alpha}, \mu_{\alpha}\right)\right\}_{\alpha \in \mathscr{A}} \subset \mathscr{C} \mathcal{M}$ is said to measured GromovHausdorff converge to $(X, \mu) \in \mathscr{C} \mathcal{M}$ if, for each $\alpha \in \mathcal{A}$, there exist $\epsilon_{\alpha}>0$ and a Borel measurable $\epsilon_{\alpha}$-approximation $\varphi_{\alpha}: X_{\alpha} \rightarrow X$ such that

(i) $\lim _{\alpha \in \mathscr{A}} \epsilon_{\alpha}=0$;

(ii) a directed system of push-forward measures $\left\{\left(\varphi_{\alpha}\right)_{*} \mu_{\alpha}\right\}_{\alpha}$ converges vaguely to $\mu$, that is,

$$
\lim _{\alpha \in \mathscr{A}} \int_{X_{\alpha}} f \circ \varphi_{\alpha} d \mu_{\alpha}=\int_{X} f d \mu
$$

holds for all continuous functions $f: X \rightarrow \mathbb{R}$.

The measured Gromov-Hausdorff convergence induces a topology on $\mathscr{G} M$. Fukaya [1987] shows it is Hausdorff [Proposition 2.7] and the projection from $\mathscr{C} \mathcal{M}$ to $\mathscr{C}$ is proper [Proposition 2.10]. Gromov [1999] gives other topologies on $\mathscr{C} . \mathcal{M}$.

A directed system $\left\{\left(X_{\alpha}, \mu_{\alpha}, x_{\alpha}\right)\right\}_{\alpha \in \mathscr{A}}$ of (possibly noncompact) pointed metric measure spaces is said to pointed measured Gromov-Hausdorff converge to a pointed metric measure space $(X, \mu, x)$ if, for any $R>0$, there exist $R_{\alpha} \searrow R$, $\epsilon_{\alpha} \searrow 0$, and Borel measurable $\epsilon_{\alpha}$-approximations $\varphi_{\alpha}: B_{R_{\alpha}}\left(x_{\alpha}\right) \rightarrow B_{R}(x)$ with $\varphi_{\alpha}\left(x_{\alpha}\right)=x$ such that the directed system $\left\{\left(\varphi_{\alpha}\right)_{*} \mu_{\alpha}\right\}_{\alpha}$ converges to $\mu$ vaguely; that is, (2-5) holds for all continuous functions $f: X \rightarrow \mathbb{R}$ with compact support.

Let $\left(M_{i}, p_{i}\right)$ for $i=1,2, \ldots$ be $n$-dimensional, complete, pointed Riemannian manifolds with $\operatorname{Ric}_{M_{i}} \geq-(n-1)$ and $v \leq \operatorname{vol}_{M_{i}}\left(B_{1}\left(p_{i}\right)\right) \leq V$ for some $0<v \leq V$. Assume that $\left\{\left(M_{i}, p_{i}\right)\right\}$ pointed Gromov-Hausdorff converges to a pointed metric space $(X, x)$. Then there exists a measure $\mu$ on $X$ such that $\left\{\left(M_{i}, \operatorname{vol}_{M_{i}}, p_{i}\right)\right\}$ pointed measured Gromov-Hausdorff subconverges to $(X, \mu, x)$. In the noncollapsed case, $\mu$ is unique and it coincides with the $n$-dimensional Hausdorff measure, up to constant multiple [Cheeger and Colding 1997, Theorem 5.9]; they show in the collapsed case that $\mu$ is not necessarily unique [Example 1.24, Section 8].

\section{The generalized Bishop-Gromov inequality}

In this section, we define the generalized Bishop-Gromov inequality $\mathrm{BG}(k, n)$ with constant $C$ and give basic properties of a metric measure space satisfying $\mathrm{BG}(k, n)$ with $C$.

We denote by $c\left(c_{1}, \ldots, c_{l}\right)$ a positive constant depending only on $c_{1}, \ldots, c_{l}$. Let $(X, d, \mu)$ be a complete, locally compact length space equipped with a Borel measure. 
3.1. Definition of the generalized Bishop-Gromov inequality. Let $k, n \in \mathbb{R}$ with $n \geq 1$. For $0 \leq r_{1}<r_{2}$, we define

$$
V_{k, n}\left(r_{1}, r_{2}\right)=\alpha_{n-1} \int_{r_{1}}^{r_{2}} \mathrm{~s}_{k}(t)^{n-1} d t
$$

where $\alpha_{n-1}:=2 \pi^{n / 2} / \Gamma(n / 2)=n \omega_{n}$ and $\mathrm{s}_{k}(t)$ for $t \geq 0$ is defined by

$$
\mathrm{s}_{k}(t)= \begin{cases}\frac{1}{\sqrt{k}} \sin (\sqrt{k} t) & \text { if } k>0, \\ t & \text { if } k=0, \\ \frac{1}{\sqrt{-k}} \sinh (\sqrt{-k} t) & \text { if } k<0 .\end{cases}
$$

If $n$ is a positive integer, then $V_{k, n}\left(r_{1}, r_{2}\right)$ is equal to the volume of an annulus of radius between $r_{1}$ and $r_{2}$ in the $n$-dimensional, complete, simply connected space of constant curvature $k$. We denote by $B_{r}(x)$ and $\bar{B}_{r}(x)$ the balls of radius $r$ centered at $x$ that are open and closed, respectively. For a point $x \in X$, let $U$ be a set in $A_{r_{1}, r_{2}}(x)$, where $A_{r_{1}, r_{2}}(x)=B_{r_{2}}(x) \backslash \bar{B}_{r_{1}}(x)$ and $A_{0, r}(x)=B_{r}(x)$. For $0 \leq s_{1}<s_{2}$ with $s_{1} \leq r_{1}$ and $s_{2} \leq r_{2}$, set

$$
S_{s_{1}, s_{2}}(x, U)=\left\{y \in A_{s_{1}, s_{2}}(x) \mid d(x, y)+d(y, z)=d(x, z) \text { for some } z \in U\right\},
$$

that is, $S_{s_{1}, s_{2}}(x, U)$ is the intersection of $A_{s_{1}, s_{2}}(x)$ and all minimal geodesics between $x$ and each point in $U$.

Definition 3.1. Let $C \geq 1$. We say that a metric measure space $(X, d, \mu)$ satisfies the generalized Bishop-Gromov inequality with constant $C$ or, for short, $\mathrm{BG}(k, n)$ with $C$, if for all points $x \in X$ and all $0 \leq r_{1}<r_{2}$ and $0 \leq s_{1}<s_{2}$ with $s_{1} \leq r_{1}$ and $s_{2} \leq r_{2}$, the inequality

$$
\frac{\mu(U)}{\mu\left(S_{s_{1}, s_{2}}(x, U)\right)} \leq C \frac{V_{k, n}\left(r_{1}, r_{2}\right)}{V_{k, n}\left(s_{1}, s_{2}\right)}
$$

holds for all Borel sets $U$ in $A_{r_{1}, r_{2}}(x)$.

$\mathrm{BG}(k, n)$ with $C=1$ induces the usual Bishop-Gromov inequality. Indeed, if we choose $r_{1}=s_{1}=0, s_{2}=r \leq R=r_{2}$, and $U=B_{R}(x)$, then (3-6) implies

$$
\frac{\mu\left(B_{R}(x)\right)}{\mu\left(B_{r}(x)\right)} \leq \frac{V_{k, n}(0, R)}{V_{k, n}(0, r)} \text {. }
$$

3.2. Examples. We recall metric measure spaces satisfying $\mathrm{BG}(k, n)$ with $C=1$, as shown in the introduction. Every $n$-dimensional, normed linear space equipped with the $n$-dimensional Lebesgue measure satisfies $\operatorname{BG}(0, n)$ with $C=1$.

Proposition 3.2. Let $X$ be an n-dimensional, normed linear space. Assume that $X$ is a Gromov-Hausdorff limit of Riemannian manifolds $M_{i}$ with Ricci curvature bounded from below. Then $X$ is isometric to $\mathbb{R}^{n}$. 
Proof. By expanding the metrics of converging manifolds, we may assume that $\operatorname{Ric}_{M_{i}} \geq-\delta_{i}$, where $\delta_{i} \rightarrow 0$. Since $X$ contains $n$ orthogonal lines, the splitting theorem (Theorem 2.1) completes the proof.

Definition 3.3 [Sturm 2006a, Definition 2.8]. A metric space $(X, d)$ is said to be nonbranching if for all four points $y, x_{0}, x_{1}, x_{2} \in X$ such that $y$ is a midpoint between $x_{0}$ and $x_{1}$ and, between $x_{0}$ and $x_{2}$, we have $x_{1}=x_{2}$. A point $y$ is called a midpoint between $x_{0}$ and $x_{1}$ if $2^{-1} d\left(x_{0}, x_{1}\right)=d\left(x_{0}, y\right)$.

A metric space $X$ is nonbranching if and only if for all two minimal geodesics $\gamma, \gamma^{\prime}:[0, l] \rightarrow X$ with $\gamma(0)=\gamma^{\prime}(0)$, we have $\inf \left\{t>0 \mid \gamma(t) \neq \gamma^{\prime}(t)\right\}=0$ or $\infty$, where inf $\varnothing:=\infty$. Each Alexandrov space with curvature bounded from below is nonbranching.

Remark 3.4. For nonbranching metric measure spaces, the curvature-dimension condition $\mathrm{CD}(K, N)$ implies the measure contraction property $\operatorname{MCP}(K, N)$; see [Sturm 2006b, Lemma 4.1, Theorem 5.4]. Moreover, $\operatorname{CD}(K, N)$ is a weaker condition than having $N$-Ricci curvature $\geq K$.

All $n$-dimensional Alexandrov spaces with curvature bounded below by $k$ that are given the $n$-dimensional Hausdorff measure $\mathscr{H}^{n}$ satisfy $\operatorname{MCP}((n-1) k, n)$; see [Ohta 2007, Proposition 2.8; Sturm 2006b, Theorem 5.7; Kuwae and Shioya 2001, Lemma 6.1]. For Riemannian manifolds, a lower bound on the sectional curvature implies the Ricci curvature is also bounded from below. However, it is an open problem whether those Alexandrov spaces satisfy $\operatorname{CD}((n-1) k, n)$ or have $n$-Ricci curvature $\geq(n-1) k$. In particular, we do not know whether all Alexandrov spaces can arise as Gromov-Hausdorff limits of Riemannian manifolds with Ricci curvature bounded from below.

Remark 3.5. Let $(X, d, \mu)$ be a measured Gromov-Hausdorff limit of those $n$-dimensional compact Riemannian manifolds $M_{i}$ with $\operatorname{Ric}_{M_{i}} \geq(n-1) k$ and equipped with the normalized Riemannian measure. Set $m=\operatorname{dim}_{H}(X)$, which is not necessarily an integer. Cheeger and Colding [1997, Conjecture 1.34] conjectured there exists a number $l$ with $m \leq l \leq n$ such that (3-6) holds for $\mu=\mathscr{H}^{m}$, $n=l$, and $C=1$.

3.3. Basic properties. We begin by recalling the definition of a doubling measure. We say that a measure $\mu$ is doubling if for all $R>0$ there exists a constant $C_{D}=$ $C_{D}(R) \geq 1$ such that $\mu\left(B_{2 r}(x)\right) \leq C_{D} \mu\left(B_{r}(x)\right)$ holds for all $x \in X$ and all $0<r \leq R$. If $\mu$ is doubling, then $X$ is proper (that is, all closed bounded subsets are compact). We note that if $(X, d, \mu)$ satisfies $\mathrm{BG}(k, n)$ with $C$, then $\mu$ is doubling.

Although the following lemmas and proposition are somewhat standard, we prove them for the completeness of this paper. 
Lemma 3.6. Let $(X, d, \mu)$ satisfy $\mathrm{BG}(k, n)$ with $C=1$ for some $k, n \in \mathbb{R}$ with $n \geq 1$. Then we have $\mu\left(S_{r}(x)\right)=0$ for all $x \in X$ and all $r>0$, where $S_{r}(x)$ is the sphere of radius $r$ and centered at $x$. In particular, if $X$ does not consist of a single point, then $\mu(\{x\})=0$ for all $x \in X$.

Proof. For a sufficiently small $\epsilon>0$, we have

$$
\begin{aligned}
\mu\left(S_{r}(x)\right) & \leq \mu\left(B_{r+\epsilon}(x)\right)-\mu\left(B_{r-\epsilon}(x)\right)=\mu\left(B_{r-\epsilon}(x)\right)\left(\frac{\mu\left(B_{r+\epsilon}(x)\right)}{\mu\left(B_{r-\epsilon}(x)\right)}-1\right) \\
& \leq \mu\left(B_{r-\epsilon}(x)\right)\left(\frac{V_{k, n}(0, r+\epsilon)}{V_{k, n}(0, r-\epsilon)}-1\right),
\end{aligned}
$$

where we have used (3-6) for the last step. Letting $\epsilon \rightarrow 0$ completes the proof.

Let $A \subset X$. We say that $A$ is convex if, for all pairs $x, y \in A$, all minimal geodesics between $x$ and $y$ are contained in $A$.

Lemma 3.7. Let $(X, d, \mu)$ satisfy $\mathrm{BG}(k, n)$ with $C$ for some $k \in \mathbb{R}, n \geq 1$, and $C \geq 1$. Then:

(1) If $k^{\prime} \leq k$ and $n^{\prime} \geq n$, then $(X, d, \mu)$ satisfies $\mathrm{BG}\left(k^{\prime}, n^{\prime}\right)$ with $C$.

(2) If $a, b>0$, then $(X, a d, b \mu)$ satisfies $\mathrm{BG}\left(k / a^{2}, n\right)$ with $C$.

(3) If $A$ is a convex subset in $X$, then $(A, d, \mu)$ also satisfies $\mathrm{BG}(k, n)$ with $C$.

Proof. Fix $0 \leq r_{1}<r_{2}$ and $0 \leq s_{1}<s_{2}$ with $s_{1} \leq r_{1}$ and $s_{2} \leq r_{2}$. (1) follows because $V_{k, n}\left(r_{1}, r_{2}\right) / V_{k, n}\left(s_{1}, s_{2}\right)$ is monotone nonincreasing in $k$ and monotone nondecreasing in $n$. We respectively denote by $A_{r_{1}, r_{2}}^{(d)}(x)$ and $S_{s_{1}, s_{2}}^{(d)}(x, U)$ the sets $A_{r_{1}, r_{2}}(x)$ and $S_{s_{1}, s_{2}}(x, U)$ with respect to a metric $d$. For all sets $U$ in $A_{r_{1}, r_{2}}^{(a d)}(x)$, we have

$$
\begin{aligned}
\frac{(b \mu)(U)}{(b \mu)\left(S_{s_{1}, s_{2}}^{(a d)}(x, U)\right)} & =\frac{\mu(U)}{\mu\left(S_{s_{1} / a, s_{2} / a}^{(d)}(x, U)\right)} \\
& \leq C \frac{V_{k, n}\left(r_{1} / a, r_{2} / a\right)}{V_{k, n}\left(s_{1} / a, s_{2} / a\right)}=C \frac{V_{k / a^{2}, n}\left(r_{1}, r_{2}\right)}{V_{k / a^{2}, n}\left(s_{1}, s_{2}\right)},
\end{aligned}
$$

which implies (2). Let $x \in A$ and $U \subset A_{r_{1}, r_{2}}(x)$. Since $A$ is convex, $S_{s_{1}, s_{2}}(x, U)$ is contained in $A$. We hence have (3).

Let $\epsilon>0$. A set $S \subset X$ is called an $\epsilon$-net if we have $d(x, S) \leq \epsilon$ for all $x \in X$. We say $S$ is $\epsilon$-separated if $d(x, y) \geq \epsilon$ for all pairs of distinct $x, y \in S$. Clearly, each maximal $\epsilon$-separated set is an $\epsilon$-net.

Proposition 3.8. Let $(X, d, \mu)$ be as in Lemma 3.7. Then we have $\operatorname{dim}_{\mathrm{H}}(X) \leq n$.

Proof. We will show that $\mathscr{H}^{n^{\prime}}(X)=0$ holds for all $n^{\prime}>n$. We may assume that $\operatorname{diam}(X)<\infty$. Let $\left\{x_{i}\right\}_{i=1}^{N}$ be a maximal $\delta$-separated set in $X$ for $\delta>0$. Then $\left\{x_{i}\right\}_{i=1}^{N}$ is a $\delta$-net. Note that there exists a constant $c(k, n)>0$ depending only on 
$k$ and $n$ such that $V_{k, n}(0, \delta) \geq c(k, n) \delta^{n}$ holds for all (sufficiently small if $k>0$ ) $\delta>0$. Let $x_{i_{0}}$ be a point at which $\mu\left(B_{\delta / 2}\left(x_{i_{0}}\right)\right)$ attains $\min _{1 \leq i \leq N} \mu\left(B_{\delta / 2}\left(x_{i}\right)\right)$. Note that $\left\{B_{\delta / 2}\left(x_{i}\right)\right\}_{i=1}^{N}$ is disjoint. Since $(X, d, \mu)$ satisfies $\operatorname{BG}(k, n)$ with $C$, we have

$$
\begin{aligned}
\mu(X) & \geq \sum_{i=1}^{N} \mu\left(B_{\delta / 2}\left(x_{i}\right)\right) \\
& \geq N \mu\left(B_{\delta / 2}\left(x_{i_{0}}\right)\right) \geq N C^{-1} \frac{V_{k, n}(0, \delta / 2)}{V_{k, n}(0, \operatorname{diam}(X))} \mu\left(B_{\operatorname{diam}(X)}\left(x_{i_{0}}\right)\right) .
\end{aligned}
$$

Hence $N \leq c(k, n) \delta^{-n}$, which implies

$$
\begin{aligned}
& \min \left\{N \in \mathbb{N} \mid X=\bigcup_{i=1}^{N} B_{\delta}\left(x_{i}\right)\right\} \\
& \quad \leq \max \left\{N \in \mathbb{N} \mid\left\{x_{i}\right\}_{i=1}^{N} \text { is a maximal } \delta \text {-separated set }\right\} \\
& \leq c(k, n) \delta^{-n} .
\end{aligned}
$$

Therefore

$$
\begin{aligned}
\mathscr{H}_{\delta}^{n^{\prime}}(X) & =\omega_{n^{\prime}} \inf \left\{\sum_{i=1}^{\infty}\left(\frac{\operatorname{diam}\left(U_{i}\right)}{2}\right)^{n^{\prime}} \mid X=\bigcup_{i=1}^{\infty} U_{i}, \operatorname{diam}\left(U_{i}\right) \leq \delta\right\} \\
& \leq \omega_{n^{\prime}} \inf \left\{\sum_{i=1}^{\infty}\left(\frac{\operatorname{diam}\left(B_{\delta / 2}\left(x_{i}\right)\right)}{2}\right)^{n^{\prime}} \mid X=\bigcup_{i=1}^{\infty} B_{\delta / 2}\left(x_{i}\right)\right\} \\
& \leq \omega_{n^{\prime}}\left(\frac{\delta}{2}\right)^{n^{\prime}} \min \left\{N \in \mathbb{N} \mid X=\bigcup_{i=1}^{N} B_{\delta / 2}\left(x_{i}\right)\right\} \\
& \leq c\left(k, n, n^{\prime}\right) \delta^{n^{\prime}-n} .
\end{aligned}
$$

Letting $\delta \rightarrow 0$ completes the proof.

\section{Local cut points}

In this section, we first prove Theorem 1.1. In Section 4.2, we recall the definition of an end and then prove Corollary 1.2. In addition, we investigate the geometric structure of the neighborhood of a local cut point and also study the structure of the accumulation of local cut points by using the idea of the proof of Theorem 1.1. We now assume that $(X, d)$ is a complete, locally compact length space.

\subsection{Local cut points.}

Definition 4.1 (local cut point [Gromov 1999, 3.32], $r$-cut point). We say that a point $x \in X$ is a local cut point if $U \backslash\{x\}$ is disconnected for some connected neighborhood $U$ of $x$. The degree of $x$, denoted by $\operatorname{deg}(x)$, is defined as the 
supremum of the number of connected components of $U \backslash\{x\}$ for all connected neighborhoods $U$ of $x$. Let $r>0$. We say that a point $x \in X$ is an $r$-cut point if these three conditions hold:

(i) $\bar{B}_{r}(x) \backslash\{x\}$ is disconnected;

(ii) the number of connected components of $\bar{B}_{r}(x) \backslash\{x\}$ is equal to $\operatorname{deg}(x)$;

(iii) $O \cap S_{r}(x)$ is nonempty for all connected components $O$ of $\bar{B}_{r}(x) \backslash\{x\}$.

If $x$ is a local cut point, then $U \backslash\{x\}$ is disconnected for every sufficiently small neighborhood $U$ of $x$. The end points in a graph (one-dimensional space) are not local cut points. An interior point in a graph is not always a local cut point; see Example 4.2 (3). We have $\operatorname{deg}(x) \geq 2$ for each local cut point $x$. Every local cut point with finite degree is an $r$-cut point for some $r>0$. Let $0<r_{1} \leq r_{2}$. If a point $x$ is an $r_{2}$-cut point, then $x$ is an $r_{1}$-cut point.

Example 4.2. (1) Consider the set $\bigcup_{i=0}^{\infty}\left\{r \exp \left(2^{-i} \pi \sqrt{-1}\right) \in \mathbb{C} \mid 0 \leq r \leq 2^{-i}\right\}$ with the induced distance [Shioya 1999, Example 9.1]. The origin is a local cut point and its degree is infinite.

(2) Consider the set $\bigcup_{i=1}^{\infty}\left\{(x, y) \in \mathbb{R}^{2} \mid x^{2}+(y-1 / i)^{2}=1 / i^{2}\right\}$ with the induced distance. The origin is a local cut point and its degree is infinite.

(3) Consider the set $\{(x, 0) \mid 0 \leq x \leq 1\} \cup\{(0, y) \mid 0 \leq y \leq 1\} \cup\left(\bigcup_{i=0}^{\infty}\left\{\left(x,-x+2^{-i}\right) \mid\right.\right.$ $\left.\left.\left.0 \leq x \leq 2^{-i}\right)\right\}\right) \subset \mathbb{R}^{2}$ with the induced distance. The origin is not a local cut point.

(4) Consider the interval $[0,1] \subset \mathbb{R}$ with the Euclidean distance. The point $1 / 3 \in$ $[0,1]$ is not an $r$-cut point for any $r>1 / 3$ because (iii) is not satisfied.

(5) Consider the set $\left\{(x, y) \mid x^{2}+y^{2}=1\right\} \cup\{(x, 0) \mid 1 \leq x\} \subset \mathbb{R}^{2}$ with the induced distance. The point $(-1,0)$ is not an $r$-cut point for any $r \geq \pi$ because (ii) is not satisfied.

Proof of Theorem 1.1. For any positive integer $d$ with $d \leq \operatorname{deg}(x)$, take a sufficiently small $r>0$. Choose any $d$ connected components $O_{1}, O_{2}, \ldots, O_{d}$ of $\bar{B}_{r}(x) \backslash\{x\}$ such that the degree of $x$ in $\bar{B}_{r}(x) \cap\left(O_{1} \cup \cdots \cup O_{d}\right)$ is equal to $d$ and $O_{i} \cap S_{r}(x)$ is nonempty for all $1 \leq i \leq d$. Let $l$ be a positive number with $0<l \leq r / 2$. For each $1 \leq i \leq d$, we choose a point $x_{i} \in O_{i}$ such that $d\left(x, x_{i}\right)=l$. See Figure 1 .

For a sufficiently small $0<\epsilon \ll l$, set $U=B_{\epsilon}(x) \cap O_{1}$. We claim that every minimal geodesic between any point in $U$ and $x_{i}(2 \leq i \leq d)$ passes through the local cut point $x$. Suppose that there exists a minimal geodesic $\gamma:[0, l] \rightarrow X$ from some point $y$ in $U$ to $x_{i}$ such that $\gamma$ does not pass through $x$. By the choice of $r$ and $l$, the point $\gamma(t)$ is not contained in $B_{r}(x)$ for some $t \in[0, l]$. Therefore

$$
d\left(y, x_{i}\right)=d(\gamma(0), \gamma(l))=l \geq t=d(y, \gamma(t)) \geq d(x, \gamma(t))-d(x, y)>r-\epsilon .
$$




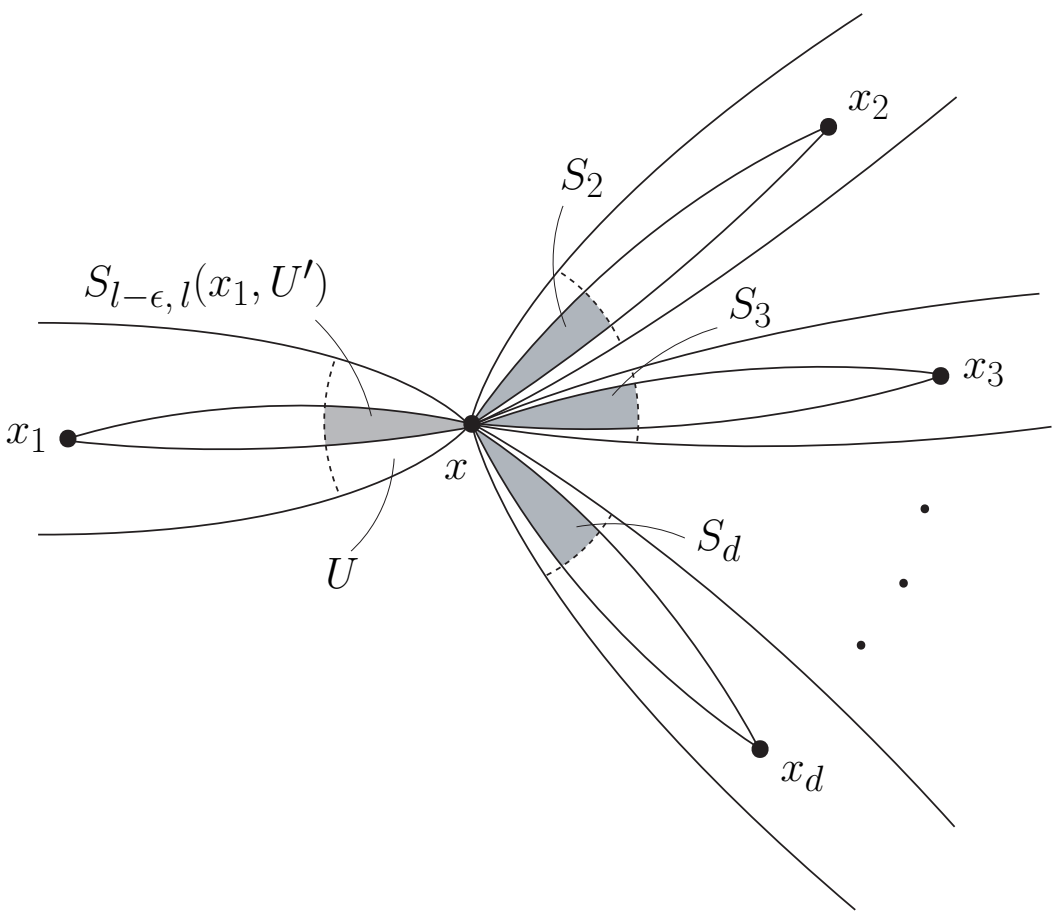

Figure 1. Proof of Theorem 1.1.

On the other hand, we have a contradiction from

$$
d\left(y, x_{i}\right) \leq d(y, x)+d\left(x, x_{i}\right)<\epsilon+l \leq \epsilon+r / 2 .
$$

We first use the generalized Bishop-Gromov inequality (3-6) with the base point $x_{i}(2 \leq i \leq d)$. We see that $A_{l, l+\epsilon}\left(x_{i}\right)$ contains $U$ for $2 \leq i \leq d$. Set $S_{i}=$ $S_{l-\epsilon, l}\left(x_{i}, U\right)$ for $2 \leq i \leq d$. We recall that $S_{i}$ is the intersection of $A_{l-\epsilon, l}\left(x_{i}\right)$ and all minimal geodesics from $x_{i}$ to each point in $U$, hence to $x$. Then $S_{i} \cap S_{j}$ is empty for all $i \neq j$. By applying (3-6) to $s_{1}=l-\epsilon, s_{2}=r_{1}=l, r_{2}=l+\epsilon$, the point $x_{i}$, and the set $U$, it follows for $2 \leq i \leq d$ that

$$
C^{-1} \frac{V_{k, n}(l-\epsilon, l)}{V_{k, n}(l, l+\epsilon)} \leq \frac{\mu\left(S_{l-\epsilon, l}\left(x_{i}, U\right)\right)}{\mu(U)}=\frac{\mu\left(S_{i}\right)}{\mu(U)} .
$$

We now denote $U^{\prime}=\bigcup_{i=2}^{d} S_{i}$. Since $\bigcap_{i=2}^{d} S_{i}=\varnothing$, we have $\sum_{i=2}^{d} \mu\left(S_{i}\right)=\mu\left(U^{\prime}\right)$. Summing up (4-7) for all $2 \leq i \leq d$, we obtain

$$
(d-1) C^{-1} \frac{V_{k, n}(l-\epsilon, l)}{V_{k, n}(l, l+\epsilon)} \leq \frac{\sum_{i=2}^{d} \mu\left(S_{i}\right)}{\mu(U)}=\frac{\mu\left(U^{\prime}\right)}{\mu(U)} .
$$


Next, we use (3-6) with the base point $x_{1}$. We see that $A_{l, l+\epsilon}\left(x_{1}\right)$ contains $U^{\prime}$. Applying (3-6) to $s_{1}=l-\epsilon, s_{2}=r_{1}=l, r_{2}=l+\epsilon$, the point $x_{1}$, and the set $U^{\prime}$, we obtain

$$
\frac{\mu\left(U^{\prime}\right)}{\mu\left(S_{l-\epsilon, l}\left(x_{1}, U^{\prime}\right)\right)} \leq C \frac{V_{k, n}(l, l+\epsilon)}{V_{k, n}(l-\epsilon, l)} .
$$

Note that, much as before, every minimal geodesic between $x_{0}$ and any point in $U^{\prime}$ passes through $x$; hence $U$ contains $S_{l-\epsilon, l}\left(x_{1}, U^{\prime}\right)$. Combining (4-8) and (4-9), we have

$$
d \leq C^{2}\left(\frac{V_{k, n}(l, l+\epsilon)}{V_{k, n}(l-\epsilon, l)}\right)^{2}+1 .
$$

Letting $\epsilon \rightarrow 0$, we have $d \leq C^{2}+1$.

For the limits of Riemannian manifolds with Ricci curvature bounded from below, the Cheeger-Colding splitting Theorem (Theorem 2.1) implies that $\operatorname{deg}(x)=2$ for any local cut point $x$; see Proposition 4.3 below. Since the splitting theorem for metric measure spaces satisfying $\operatorname{BG}(0, n)$ with $C$ do not hold in general (see Proposition 3.2), the proof of Theorem 1.1 gives another proof that $\operatorname{deg}(x)=2$.

Proposition 4.3. Let $(X, d)$ be a Gromov-Hausdorff limit of n-dimensional, complete Riemannian manifolds $\left(M_{i}, d_{i}\right)$ with $\operatorname{Ric}_{M_{i}} \geq-(n-1)$, where $d_{i}$ is the Riemannian distance on $M_{i}$. If there exists a local cut point $x$ in $X$, then every tangent cone at $x$ is isometric to $\mathbb{R} ;$ in particular, $\operatorname{deg}(x)=2$.

Proof. Take points $p_{i}$ in $M_{i}$ such that $\left\{\left(M_{i}, d_{i}, p_{i}\right)\right\}$ pointed Gromov-Hausdorff converges to $(X, d, x)$. Let us recall that a tangent cone at $x$ is the pointed limit space of $\left(X, r_{i}^{-1} d, x\right)$ as $r_{i} \rightarrow 0$. By passing to a subsequence, the tangent cone at $x$ is itself the pointed limit of rescaled manifolds $\left(M_{j}, r_{j}^{-1} d_{j}, p_{j}\right)$. Then the Ricci curvature of $\left(M_{j}, r_{j}^{-1} d_{j}\right)$ is bounded below by $-(n-1) r_{j}^{2}$.

Because every local cut point is an interior point of some geodesic, the tangent cone at $x$ contains a straight line (expanded from the geodesic). Applying the splitting theorem (Theorem 2.1), we see that the tangent cone at $x$ is isometric to $\mathbb{R} \times Y$ for some length space $Y$. Since $x$ is a local cut point, it follows that $Y$ consists of a single point.

Remark 4.4. We recall that if $(X, d, \mu)$ satisfies $\operatorname{BG}(k, n)$ with $C$, then $\mu$ is doubling. Although the length space in Example 4.2 (1) has the natural doubling measure, the degree of the origin is infinite.

In the case of graphs, we obtain a better estimate of the degree.

Proposition 4.5. Let $\left(X, d, \mathscr{H}^{1}\right)$ be a graph, where $d$ is the usual distance and $\mathscr{H}^{1}$ is the one-dimensional Hausdorff measure. Assume that $\left(X, d, \mathscr{H}^{1}\right)$ satisfies 
$\mathrm{BG}(k, n)$ with $C$ for some $k \in \mathbb{R}, n \geq 1$, and $C \geq 1$. Then we have $\operatorname{deg}(x) \leq C+1$ for all $x \in X$.

Proof. Let $x$ be a local cut point (an interior point) in $X$. Take a point $x_{1} \in X$ with $d\left(x, x_{1}\right) \ll 1$, and let $l=d\left(x, x_{1}\right)$. Let $\gamma:[0, l] \rightarrow X$ be the minimal geodesic from $x_{1}$ to $x$. Set $U=B_{\epsilon}(x) \backslash \gamma([0, l])$ for $0<\epsilon \ll 1$. We then have $S_{l-\epsilon, l}\left(x_{1}, U\right)=$ $\gamma((l-\epsilon, l))$ because $l=d\left(x, x_{1}\right)$ is sufficiently small. By using (3-6) with $s_{1}=l-\epsilon$, $s_{2}=r_{1}=l, r_{2}=l+\epsilon$, the point $x_{1}$, and the set $U$, it follows that

$$
\frac{(\operatorname{deg}(x)-1) \epsilon}{\epsilon}=\frac{\mathscr{H}^{1}(U)}{\mathscr{H}^{1}\left(S_{l-\epsilon, l}\left(x_{1}, U\right)\right)} \leq C \frac{V_{k, n}(l, l+\epsilon)}{V_{k, n}(l-\epsilon, l)} .
$$

Taking $\epsilon \rightarrow 0$, we obtain $\operatorname{deg}(x) \leq C+1$.

Remark 4.6. In general, the converse of Proposition 4.5 does not necessarily hold.

4.2. The number of ends. We recall the definition of an end.

Definition 4.7. A path $\gamma:[0, \infty) \rightarrow X$ is called a ray if (2-4) holds for all $t, t^{\prime} \in$ $[0, \infty)$. Let $\gamma_{1}, \gamma_{2}:[0, \infty) \rightarrow X$ be rays from the base point $x$. Two rays $\gamma_{1}$ and $\gamma_{2}$ are said to be cofinal if $\gamma_{1}(t)$ and $\gamma_{2}(t)$ lie in the same connected component of $X \backslash B_{r}(x)$ for all $t, r>0$ with $t \geq r$. An equivalence class of cofinal rays is called an end of $X$.

Proof of Corollary 1.2. Suppose that the number of ends of $X$ is greater than $C^{2}+1$. Given any sequence $\epsilon_{i} \rightarrow 0$ and any point $x \in X$, we put $\left(X_{i}, d_{i}, x_{i}\right)=\left(X, \epsilon_{i} d, x\right)$. Let $\mu_{i}$ be the push-forward of the measure $\mu\left(B_{1 / \epsilon_{i}}(x)\right)^{-1} \mu$ by the identity map from $X$ to $X_{i}$. From Lemma 3.7 (2) the metric measure space $\left(X_{i}, d_{i}, \mu_{i}\right)$ satisfies $\operatorname{BG}(0, n)$ with $C$. Then there is a subsequence $\epsilon_{j} \rightarrow 0$ such that $\left\{\left(X_{j}, d_{j}, \mu_{j}, x_{j}\right)\right\}$ pointed measured Gromov-Hausdorff converges to some pointed metric measure space $\left(X_{\infty}, d_{\infty}, \mu_{\infty}, x_{\infty}\right)$. The limit space also satisfies $\operatorname{BG}(0, n)$ with $C$. Since the number of ends of $X$ is greater than two, the point $x_{\infty}$ is a local cut point. Then the degree of $x_{\infty}$ is equal to the number of ends of $X$. This contradicts Theorem 1.1 .

4.3. Branch points. We next give an obstruction condition for the existence of a local cut point in a metric measure space $X$ satisfying $\operatorname{BG}(k, n)$ with $1 \leq C<\sqrt{2}$. Let us recall that the measured Gromov-Hausdorff limits of manifolds $M_{i}$ with $\operatorname{Ric}_{M_{i}} \geq(n-1) k$ satisfy $\operatorname{BG}(k, n)$ with $C=1$. It is conjectured that the limit space has no local cut point unless the limit is one-dimensional.

Assume that $X$ has a local cut point $x$. Let $x$ be an $r$-cut point. Let $\gamma:[0, l] \rightarrow X$ be a minimal geodesic with $\gamma(l)=x$. Assume that $l$ is sufficiently small (for example, $l \leq r / 3)$. If $\sigma:[0, L] \rightarrow X$ is a minimal geodesic such that $\sigma(0)=\gamma(0)$ and $\sigma(L) \in B_{l}(\gamma(l))$ for $L \geq l$, then $\sigma(l)=\gamma(l)$, that is, $\sigma$ passes through $x$. We then define two kinds of branch points of the geodesic $\gamma$. 


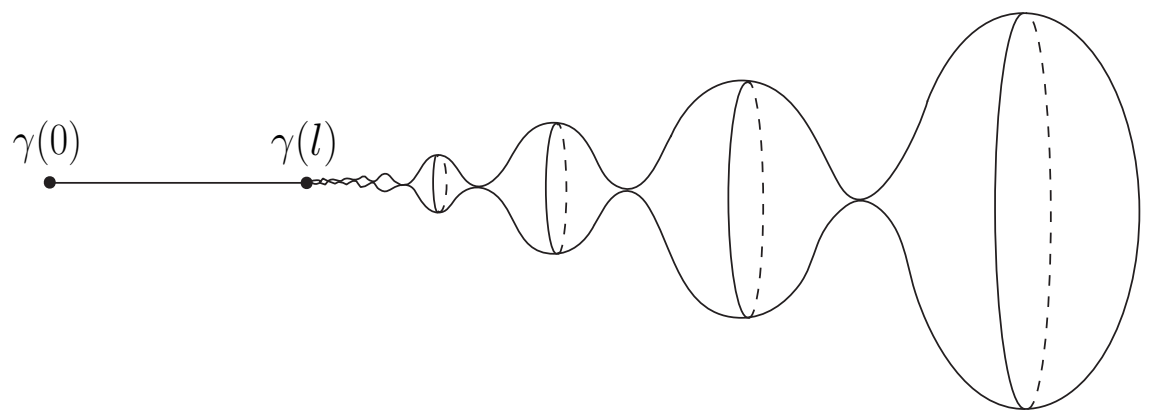

Figure 2. Weak branch point.

Definition 4.8 (branch point). Let $\gamma$ be as above. We say that $\gamma(l)$ is a branch point of $\gamma$ if for all $\epsilon>0$ there exist two distinct points $x_{1}, x_{2} \in B_{\epsilon}(\gamma(l))$ such that $d\left(\gamma(0), x_{1}\right)=d\left(\gamma(0), x_{2}\right)>l$.

Note that $\gamma(l)$ is a branch point of $\gamma$ if and only if no neighborhood of $\gamma(l)$ is a segment.

Definition 4.9 (weak branch point [Cheeger and Colding 2000a, Section 5]). Let $\gamma$ be as in Definition 4.8. We say that $\gamma(l)$ is a weak branch point of $\gamma$ if, for all $\epsilon>0$ and all two points $x_{1}, x_{2} \in B_{\epsilon}(\gamma(l))$ with $d\left(\gamma(0), x_{1}\right), d\left(\gamma(0), x_{2}\right)>l$, there exist minimal geodesics $\sigma_{i}:\left[0, l_{i}\right] \rightarrow X$ for $i=1,2$ from $\gamma(l)$ to $x_{i}$ such that $\sigma_{1}(s)=\sigma_{2}(s)$ for some $s>0$. See Figure 2 .

Example 4.10. (1) Let $X=[a, b]$. Every interior point $x \in(a, b)$ is a weak branch point of all paths $\gamma:[0, l] \rightarrow X$ with $\gamma(l)=x$ and $l \ll 1$. The point $x$ is not a branch point of any $\gamma$.

(2) Let $X$ be the length space in Figure 2. The intersection $x$ of two spheres is a branch point of all paths $\gamma:[0, l] \rightarrow X$ with $\gamma(l)=x$ and $l \ll 1$. However, the point $x$ is not a weak branch point. Although a branch point is not necessarily a weak branch point, we use the term "weak" as in [Cheeger and Colding 2000a, Section 5].

Assume now that there exists a local cut point in a metric measure space satisfying $\mathrm{BG}(k, n)$ with $1 \leq C<\sqrt{2}$. We give an obstruction condition for the existence of a local cut point by observing geodesics that pass through a local cut point.

Theorem 4.11 [Cheeger and Colding 2000a, Theorem 5.1]. Let $(X, d, \mu)$ be a metric measure space satisfying the generalized Bishop-Gromov inequality $\mathrm{BG}(k, n)$ with constant $C$ for some $k \in \mathbb{R}, n \geq 1$, and $1 \leq C<\sqrt{2}$. If there exists a local cut point $x$ in $X$, then it is a weak branch point of all geodesics $\gamma:[0, l] \rightarrow X$ with $\gamma(l)=x$ and $l \ll 1$. 
The proof is essentially the same as in [Cheeger and Colding 2000a]. We give the proof for the completeness of the paper.

Proof. If $x$ has a neighborhood that is a segment, then $x$ is a weak branch point of all paths which go to the point. Assume now that no neighborhood of $x$ is a segment. Let $\gamma:[0, l] \rightarrow X$ be a geodesic that branches at $\gamma(l)=x$, where $l$ is sufficiently small. Suppose that $x$ is not a weak branch point: There exist two points $x_{1}, x_{2} \in B_{l}(\gamma(l))$ with $d\left(\gamma(0), x_{1}\right), d\left(\gamma(0), x_{2}\right)>l$ such that all geodesics $\sigma_{i}:\left[0, l_{i}\right] \rightarrow X$ for $i=1,2$ from $\gamma(l)$ to $x_{i}$ have $\sigma_{1}(s) \neq \sigma_{2}(s)$ for all $0<s \leq$ $\min \left\{l_{1}, l_{2}\right\}$. We may assume that $d\left(\gamma(l), x_{1}\right)=d\left(\gamma(l), x_{2}\right)$ and denote both by $l^{\prime}$.

Consider all minimal geodesics from $x$ to $x_{i}$ for $i=1,2$. By assumption, the set of all minimal geodesics from $x$ to $x_{1}$ and the set of all minimal geodesics from $x$ to $x_{2}$ have no intersection except $x$. We will use the generalized BishopGromov inequality (3-6) with the base point $x_{i}$. Let $0<\epsilon \ll l^{\prime}$. We denote by $U$ the connected component of $B_{\epsilon}(x) \backslash\{x\}$ that contains $\gamma$. We see that $A_{l^{\prime}, l^{\prime}+\epsilon}\left(x_{i}\right)$ contains $U$. Set $S_{i}=S_{l^{\prime}-\epsilon, l^{\prime}}\left(x_{i}, U\right)$ for $i=1,2$. Let us recall that $S_{i}$ is the intersection of $A_{l^{\prime}-\epsilon, l^{\prime}}\left(x_{i}\right)$ and all minimal geodesics from $x_{i}$ to each point in $U$. Then $S_{1} \cap S_{2}$ is empty by assumption. For $i=1,2$, the generalized Bishop-Gromov inequality (3-6) induces

$$
C^{-1} \frac{V_{k, n}\left(l^{\prime}-\epsilon, l^{\prime}\right)}{V_{k, n}\left(l^{\prime}, l^{\prime}+\epsilon\right)} \leq \frac{\mu\left(S_{l^{\prime}-\epsilon, l^{\prime}}\left(x_{i}, U\right)\right)}{\mu(U)}=\frac{\mu\left(S_{i}\right)}{\mu(U)} .
$$

Let $U^{\prime}$ denote the union of connected components of $B_{\epsilon}(x) \backslash\{x\}$ that contain $S_{1}$ and $S_{2}$. We see that $A_{l, l+\epsilon}(\gamma(0))$ contains $U^{\prime}$. Since $S_{i} \subset U^{\prime}$ holds for $i=1,2$, we have $\mu\left(S_{1}\right)+\mu\left(S_{2}\right) \leq \mu\left(U^{\prime}\right)$. By summing up (4-11) for $i=1,2$, it follows that

$$
2 C^{-1} \frac{V_{k, n}\left(l^{\prime}-\epsilon, l^{\prime}\right)}{V_{k, n}\left(l^{\prime}, l^{\prime}+\epsilon\right)} \leq \frac{\mu\left(S_{1}\right)+\mu\left(S_{2}\right)}{\mu(U)} \leq \frac{\mu\left(U^{\prime}\right)}{\mu(U)} .
$$

Applying (3-6) with the base point $\gamma(0)$, we obtain

$$
\frac{\mu\left(U^{\prime}\right)}{\mu\left(S_{l-\epsilon, l}\left(\gamma(0), U^{\prime}\right)\right)} \leq C \frac{V_{k, n}(l, l+\epsilon)}{V_{k, n}(l-\epsilon, l)} .
$$

Since $U$ contains $S_{l-\epsilon, l}\left(\gamma(0), U^{\prime}\right)$, it, together with (4-12) and (4-13), follows that

$$
2 C^{-1} \frac{V_{k, n}\left(l^{\prime}-\epsilon, l^{\prime}\right)}{V_{k, n}\left(l^{\prime}, l^{\prime}+\epsilon\right)} \leq C \frac{V_{k, n}(l, l+\epsilon)}{V_{k, n}(l-\epsilon, l)} .
$$

Taking $\epsilon \rightarrow 0$, we obtain $2 \leq C^{2}$, which is a contradiction.

Remark 4.12. Cheeger and Colding [2000a, Theorem 5.1] studied the limit space that contains a one-dimensional piece and is not one-dimensional. It follows from Theorem 4.11 that the length space in Figure 2 can't satisfy $\mathrm{BG}(k, n)$ with $1 \leq C<$ $\sqrt{2}$ for any $k, n$, and any measure. This does not follow from their [Theorem 5.1]. 


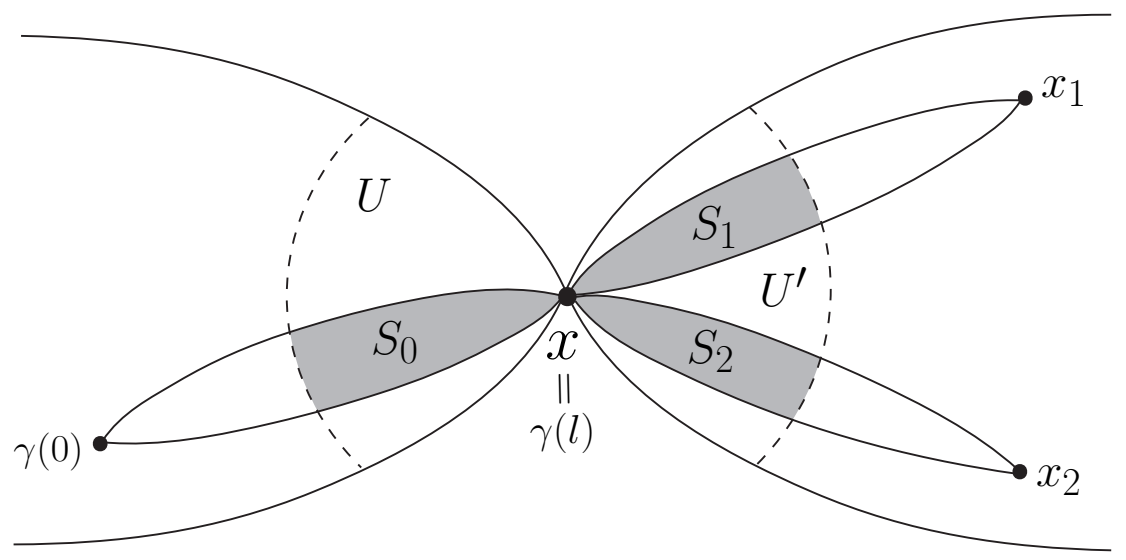

Figure 3. Proof of Theorem 4.11.

As a corollary of Theorem 4.11, we obtain a particular case of Theorem 1.1.

Theorem 4.13. Let $(X, d, \mu)$ satisfy $\mathrm{BG}(k, n)$ with $C$ for some $k \in \mathbb{R}, n \geq 1$, and $1 \leq C<\sqrt{2}$. Assume that there exists a local cut point $x$ in $X$. Then $\operatorname{deg}(x)=2$.

Proof. Suppose that $\operatorname{deg}(x) \geq 3$. For all geodesics $\gamma:[0, l] \rightarrow X$ with $\gamma(l)=x$ and $l \ll 1$, the local cut point $x=\gamma(l)$ is not a weak branch point of $\gamma$. This is because the geodesic $\gamma$ is extended to two connected components of $B_{r}(x) \backslash\{x\}$ which do not contain $\gamma$, where $r$ is sufficiently small. This contradicts Theorem 4.11.

4.4. Local geometric structure. Recall the definition of an $r$-cut point (Definition 4.1). We investigate the geometric structure of the neighborhood of an $r$-cut point in a metric measure space $X$ satisfying $\mathrm{BG}(k, n)$ with $1 \leq C<\sqrt{2}$.

Assume $X$ has an $r$-cut point $x$. Then $\operatorname{diam}\left(O \cap S_{r}(x)\right) \leq \operatorname{diam}\left(\bar{B}_{r}(x)\right) \leq 2 r$ for all connected components $O$ of $\bar{B}_{r}(x) \backslash\{x\}$; see Figure 5. By improving the method of the proof of Theorem 4.11, we obtain a more precise estimate of the diameter:

Theorem 4.14. For all $k \in \mathbb{R}, n \geq 1,1 \leq C<\sqrt{2}$, and all $R>0$ there exists $a$ constant $\delta=\delta(k, n, C, R)>0$ depending only on $k, n, C$, and $R$ such that: Let $(X, d, \mu)$ be a metric measure space satisfying the generalized Bishop-Gromov inequality $\mathrm{BG}(k, n)$ with constant $C$. If $X$ has an $r$-cut point $x$ with $0<r \leq R$, then $\operatorname{diam}\left(O \cap S_{r}(x)\right) \leq(2-\delta) r$ holds for all connected components $O$ of $\bar{B}_{r}(x) \backslash\{x\}$.

Remark 4.15. The constant $\delta(k, n, C, R)$ in Theorem 4.14 is independent of the metric measure space $(X, d, \mu)$. Moreover, we can calculate the precise value; see Remark 4.19. It follows from Theorem 4.14 that for fixed $k, n$, and $C$ the metric space in Figure 4 cannot satisfy $\mathrm{BG}(k, n)$ with $C$ for any measure, provided 


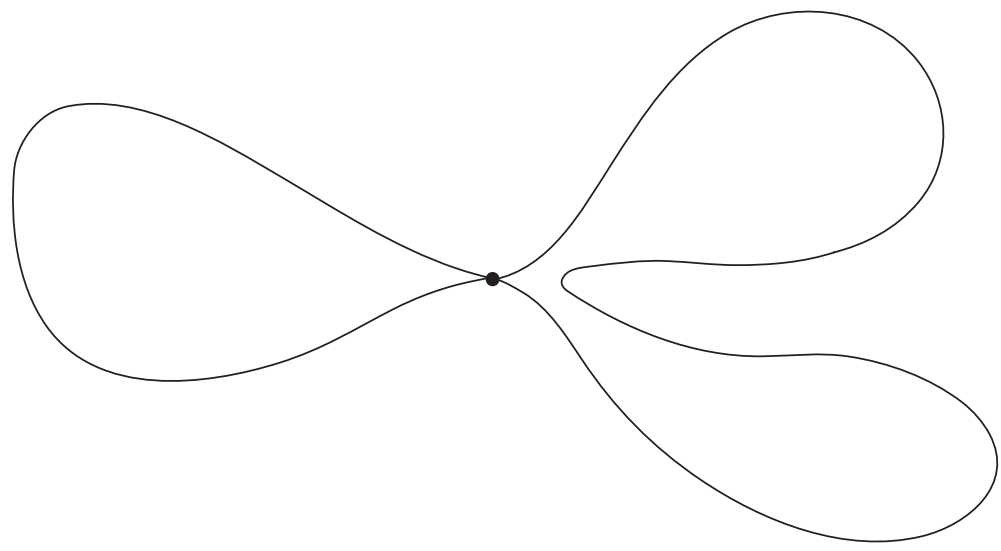

Figure 4. "Three-pronged" space.

a narrow part is sufficiently small. Roughly speaking, combined with Theorem 4.13, "three-pronged" spaces cannot satisfy $\mathrm{BG}(k, n)$ with $1 \leq C<\sqrt{2}$.

Proof of Theorem 4.14. For $k \in \mathbb{R}, n \geq 1,1 \leq C<\sqrt{2}$, and $R>0$, let $(X, d, \mu)$ be a metric measure space satisfying $\mathrm{BG}(k, n)$ with $C$ and $x \in X$ be an $r$-cut point with $0<r \leq R$. Fix $0<\delta \ll r$. Suppose that diam $\left(O \cap S_{r}(x)\right)>(2-\delta) r$ holds for some connected component $O$ of $\bar{B}_{r}(x) \backslash\{x\}$. We take two points $x_{1}, x_{2} \in O \cap S_{r}(x)$ with $d\left(x_{1}, x_{2}\right)>(2-\delta) r$ and then choose minimal geodesics $\sigma_{i}:[0, r] \rightarrow X$ from $x$ to $x_{i}$ for $i=1,2$. Since $\operatorname{deg}(x)=2$, we denote by $O^{\prime}$ another connected component of $\bar{B}_{r}(x) \backslash\{x\}$. For $0<\epsilon<\delta r$, denote by $U$ the connected component of $B_{\epsilon}(x) \backslash\{x\}$ that does not contain $\sigma_{1}$ and $\sigma_{2}$. Then $A_{r / 3, r / 3+\epsilon}\left(\sigma_{i}(r / 3)\right)$ contains $U$ for $i=1,2$.

Claim 4.16. Every minimal geodesic from $\sigma_{i}(r / 3)$ to a point in $U$ passes through $x$ for $i=1,2$.

Proof. Suppose that there exists a minimal geodesic $\gamma:[0, l] \rightarrow X$ from $\sigma_{i}(r / 3)$ to some point $y$ in $U$ such that $\gamma$ does not pass through $x$. Then, $\gamma(t) \notin B_{r}(x)$ for some $t \in[0, l]$ since $x$ is an $r$-cut point. Therefore,

$$
d\left(y, \sigma_{i}(r / 3)\right) \geq d(y, \gamma(t)) \geq d(\gamma(t), x)-d(y, x)>r-\epsilon .
$$

On the other hand, we have a contradiction from

$$
d\left(y, \sigma_{i}(r / 3)\right) \leq d(y, x)+d\left(x, \sigma_{i}(r / 3)\right)<\epsilon+r / 3 .
$$

Setting $S_{i}=S_{r / 3-\delta r-\epsilon, r / 3-\delta r}\left(\sigma_{i}(r / 3), U\right)$ for $i=1,2$, we have

Claim 4.17. $S_{1} \cap S_{2}=\varnothing$. 
Proof. By assumption, we have

$$
\begin{aligned}
(2-\delta) r<d\left(x_{1}, x_{2}\right) & \leq d\left(x_{1}, \sigma_{1}(r / 3)\right)+d\left(\sigma_{1}(r / 3), \sigma_{2}(r / 3)\right)+d\left(\sigma_{2}(r / 3), x_{2}\right) \\
& =\frac{2 r}{3}+d\left(\sigma_{1}(r / 3), \sigma_{2}(r / 3)\right)+\frac{2 r}{3} .
\end{aligned}
$$

It follows that $(2 / 3-\delta) r<d\left(\sigma_{1}(r / 3), \sigma_{2}(r / 3)\right)$. Suppose that there exists a point $y \in S_{1} \cap S_{2}$. We then have

$$
d\left(\sigma_{1}(r / 3), \sigma_{2}(r / 3)\right) \leq d\left(\sigma_{1}(r / 3), y\right)+d\left(y, \sigma_{2}(r / 3)\right) \leq 2\left(\frac{r}{3}-\delta r\right)=\left(\frac{2}{3}-2 \delta\right) r .
$$

This is a contradiction.

The generalized Bishop-Gromov inequality (3-6) with the base point $\sigma_{i}(r / 3)$ gives, for $i=1,2$,

$$
C^{-1} \frac{V_{k, n}((1 / 3-\delta) r-\epsilon,(1 / 3-\delta) r)}{V_{k, n}(r / 3, r / 3+\epsilon)} \leq \frac{\mu\left(S_{i}\right)}{\mu(U)} .
$$

We set $U^{\prime}=S_{1} \cup S_{2}$. Then $S_{1} \cap S_{2}=\varnothing$ (Claim 4.17) gives $\mu\left(S_{1}\right)+\mu\left(S_{2}\right) \leq \mu\left(U^{\prime}\right)$. Therefore, by summing (4-14) for $i=1,2$, we get

$$
2 C^{-1} \frac{V_{k, n}((1 / 3-\delta) r-\epsilon,(1 / 3-\delta) r)}{V_{k, n}(r / 3, r / 3+\epsilon)} \leq \frac{\mu\left(S_{1}\right)+\mu\left(S_{2}\right)}{\mu(U)} \leq \frac{\mu\left(U^{\prime}\right)}{\mu(U)} .
$$

Next, we take an arbitrary point $x_{0}$ in $O^{\prime} \cap S_{r / 3}(x)$. Such a point exists since $x$ is an $r$-cut point.

Claim 4.18. Each minimal geodesic from $x_{0}$ to each point in $U^{\prime}$ passes through $x$.

Proof. Suppose that there exists a minimal geodesic $\gamma:[0, l] \rightarrow X$ from $x_{0}$ to some point $y$ in $U^{\prime}$ such that $\gamma$ does not pass through $x$. Then $\gamma(t) \notin B_{r}(x)$ for some $t \in[0, l]$. Therefore,

$$
d\left(x_{0}, y\right) \geq d\left(x_{0}, \gamma\left(t_{0}\right)\right) \geq d\left(\gamma\left(t_{0}\right), x\right)-d\left(x, x_{0}\right) \geq r-\frac{r}{3}=\frac{2 r}{3} .
$$

On the other hand, we have a contradiction from

$$
d\left(x_{0}, y\right) \leq d\left(x_{0}, x\right)+d(x, y) \leq \frac{r}{3}+\delta r+\epsilon .
$$

Set $S_{0}=S_{r / 3-\epsilon, r / 3}\left(x_{0}, U^{\prime}\right)$. The generalized Bishop-Gromov inequality (3-6) with the base point $x_{0}$ implies

$$
\frac{\mu\left(U^{\prime}\right)}{\mu\left(S_{0}\right)} \leq C \frac{V_{k, n}((1 / 3+\delta) r,(1 / 3+\delta) r+\epsilon)}{V_{k, n}(r / 3-\epsilon, r / 3)} .
$$




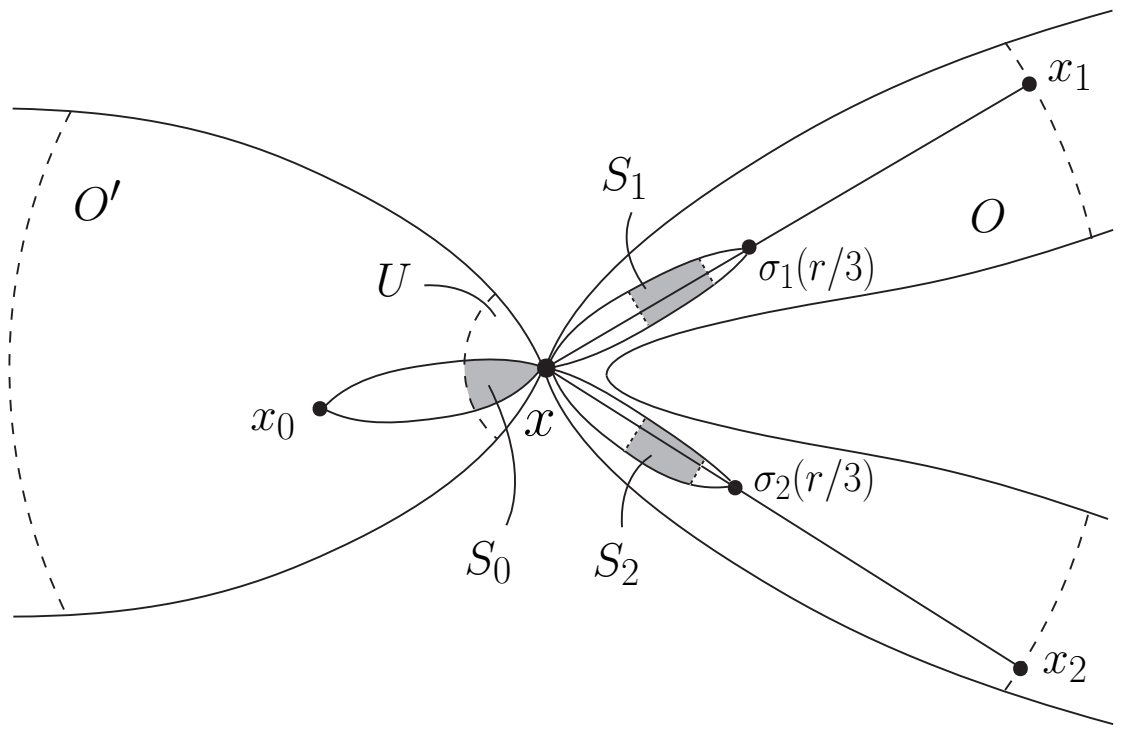

Figure 5. Proof of Theorem 4.14.

Since $U$ contains $S_{0}$, inequalities (4-15) and (4-16) give

$$
2 \leq C^{2} \frac{V_{k, n}(r / 3, r / 3+\epsilon)}{V_{k, n}(r / 3-\epsilon, r / 3)} \frac{V_{k, n}((r / 3+\delta) r,(r / 3+\delta) r+\epsilon)}{V_{k, n}((r / 3-\delta) r-\epsilon,(r / 3-\delta) r)} .
$$

After letting $\epsilon \rightarrow 0$, a sufficiently small $\delta>0$ implies a contradiction. This completes the proof of Theorem 4.14.

Remark 4.19. In the case $k=0$, we have

$$
\delta(0, n, C, R)=\frac{1}{4} \frac{\left(2 / C^{2}\right)^{1 /(n-1)}-1}{\left(2 / C^{2}\right)^{1 /(n-1)}+1} .
$$

Indeed, the right-hand side of (4-17) is equal to

$$
C^{2} \frac{(r / 3+\epsilon)^{n}-(r / 3)^{n}}{(r / 3)^{n}-(r / 3-\epsilon)^{n}} \frac{[(1 / 3+\delta) r+\epsilon]^{n}-[(1 / 3+\delta) r]^{n}}{[(1 / 3-\delta) r]^{n}-[(1 / 3-\delta) r-\epsilon]^{n}},
$$

which converges to $C^{2}[(1 / 3+\delta) /(1 / 3-\delta)]^{n-1}$ as $\epsilon \rightarrow 0$. Therefore, it suffices to determine a positive number $\delta$ such that $C^{2}[(1 / 3+\delta) /(1 / 3-\delta)]^{n-1}<2$.

4.5. Convergence of local cut points. Using Theorem 4.14, we observe the structure of the accumulation of local cut points in a metric measure space satisfying $\mathrm{BG}(k, n)$ with $1 \leq C<\sqrt{2}$.

Let $(X, d, \mu)$ satisfy $\mathrm{BG}(k, n)$ with $C$ for some $k \in \mathbb{R}, n \geq 1$, and $1 \leq C<$ $\sqrt{2}$. Assume that there exist three $r$-cut points $x_{1}, x_{2}, x_{3} \in X(r>0)$ such that 
$d\left(x_{i}, x_{j}\right) \ll r$ for all $1 \leq i, j \leq 3$. We recall that $\operatorname{deg}\left(x_{i}\right)=2$ (Theorem 4.13). Denote by $O_{1}, O_{1}^{\prime}$ the connected components of $\bar{B}_{r}\left(x_{1}\right) \backslash\left\{x_{1}\right\}$. Assume then that $O_{1}^{\prime}$ contains $x_{2}$ and $x_{3}$ and without loss of generality that $d\left(x_{1}, x_{2}\right)<d\left(x_{1}, x_{3}\right)$. For $i=2,3$, denote by $O_{i}$ the connected component of $\bar{B}_{r}\left(x_{i}\right) \backslash\left\{x_{i}\right\}$ that contains $x_{1}$ and by $O_{i}^{\prime}$ the connected component of $\bar{B}_{r}\left(x_{i}\right) \backslash\left\{x_{i}\right\}$ that does not contain $x_{1}$. By definition, $O_{2} \cap O_{3}$ is nonempty. We say that $\left\{x_{1}, x_{2}, x_{3}\right\}$ stands in a line if $\mathrm{O}_{2}^{\prime} \cap \mathrm{O}_{3}^{\prime}$ is nonempty.

As a corollary of Theorem 4.14, we have

Corollary 4.20. Let $(X, d, \mu)$ be a metric measure space satisfying $\mathrm{BG}(k, n)$ with $C$ for some $k \in \mathbb{R}, n \geq 1$, and $1 \leq C<\sqrt{2}$. Let $\delta=\delta(k, n, C, R)$ be the constant in Theorem 4.14 for $R>0$. Assume that there exist $r$-cut points $x_{1}, x_{2}, x_{3} \in X$ with $0<r \leq R$. If $d\left(x_{i}, x_{j}\right)<\delta r / 6$ holds for all $1 \leq i, j \leq 3$, then $\left\{x_{1}, x_{2}, x_{3}\right\}$ stands in a line.

Proof. Suppose that $O_{2}^{\prime} \cap O_{3}^{\prime}$ is empty; see Figure 6. Since $x_{2}$ is an $r / 3$-cut point, $O_{2} \cap S_{r / 3}\left(x_{2}\right)=\left(O_{1} \cap S_{r / 3}\left(x_{2}\right)\right) \cup\left(O_{3}^{\prime} \cap S_{r / 3}\left(x_{2}\right)\right)$ has at least two connected components. Denote by $O$ one of connected components of $O_{1} \cap S_{r / 3}\left(x_{2}\right)$ and by $O^{\prime}$ one of connected components of $O_{3}^{\prime} \cap S_{r / 3}\left(x_{2}\right)$.

Since $x_{2}$ is an $r$-cut point, all minimal geodesics from each point in $O$ to each point in $O^{\prime}$ pass through $x_{1}$ and $x_{3}$. Therefore, we have

$$
d\left(O, O^{\prime}\right)=d\left(x_{1}, O\right)+d\left(x_{1}, x_{3}\right)+d\left(x_{3}, O^{\prime}\right) .
$$

Since $d\left(x_{i}, x_{j}\right)<\delta r / 6$ holds by the assumption, we have

$$
d\left(x_{1}, O\right) \geq \frac{r}{3}-d\left(x_{2}, x_{1}\right)>\frac{r}{3}-\frac{\delta r}{6}=\left(1-\frac{\delta}{2}\right) \frac{r}{3} .
$$

Similarly, we have

$$
d\left(x_{3}, O^{\prime}\right)>\left(1-\frac{\delta}{2}\right) \frac{r}{3} .
$$

Hence, relations (4-18), (4-19) and (4-20) imply

$$
\begin{aligned}
\operatorname{diam}\left(O_{2} \cap S_{r / 3}\left(x_{2}\right)\right) & \geq d\left(O, O^{\prime}\right)=d\left(x_{1}, O\right)+d\left(x_{1}, x_{3}\right)+d\left(x_{3}, O^{\prime}\right) \\
& >\left(1-\frac{\delta}{2}\right) \frac{r}{3}+\left(1-\frac{\delta}{2}\right) \frac{r}{3} \\
& =(2-\delta) \frac{r}{3} .
\end{aligned}
$$

This contradicts Theorem 4.14.

Assume now that there exists a sequence $\left\{x_{i}\right\}_{i=1}^{\infty}$ of $r$-cut points in $X$ such that $d\left(x_{i}, x_{j}\right) \ll r$ for all $i$ and $j$. Denote by $O_{i}$ and $O_{i}^{\prime}$ the connected components of $\bar{B}_{r}\left(x_{i}\right) \backslash\left\{x_{i}\right\}$. Assume that $O_{i}$ contains $x_{j}$ for all $j<i$. We say that $\left\{x_{i}\right\}_{i=1}^{\infty}$ stands in a line if $O_{i}^{\prime} \cap O_{i+1}^{\prime}$ is nonempty for all $i$. Corollary 4.20 implies: 


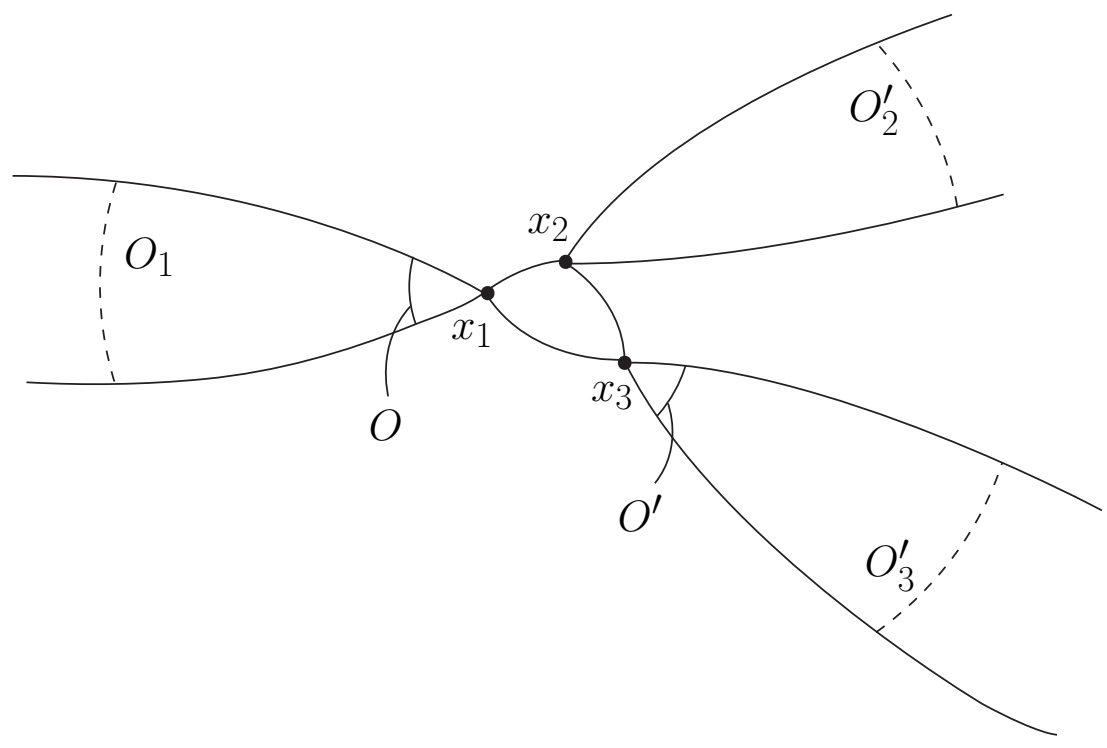

Figure 6. Proof of Corollary 4.20.

Corollary 4.21. Let $(X, d, \mu)$ be as in Corollary 4.20. For each $r>0$, the set of all $r$-cut points in $X$ is closed.

Proof. Assume that there exists a sequence $\left\{x_{i}\right\}_{i=1}^{\infty}$ of $r$-cut points in $X$ that converges to a point $x$ in $X$. By Corollary 4.20, $\left\{x_{i}\right\}_{i=N}^{\infty}$ stands in a line for a sufficiently large $N$. Hence the limit $x$ is a local cut point. Since each $x_{i}$ is an $r$-cut point, so is $x$.

Remark 4.22. Let $\left\{x_{i}\right\}_{i=1}^{\infty}$ be a sequence of $r_{i}$-cut points, where $r_{i} \rightarrow 0$. It is then not necessarily true that a limit point of $\left\{x_{i}\right\}$ is a local cut point. Consider the sequence $\{1 / i\}_{i=1}^{\infty} \subset[0,1]$.

In fact, Corollary 4.21 holds without the assumption $\mathrm{BG}(k, n)$ with $1 \leq C<\sqrt{2}$.

Proposition 4.23. Let $(X, d)$ be a complete, locally compact length space. Assume that for $r>0$ there exists a sequence $\left\{x_{i}\right\}_{i=1}^{\infty}$ of $r$-cut points in $X$ that converges to a point $x \in X$. Then, for some sufficiently large $N$, we have $\operatorname{deg}\left(x_{i}\right)=2$ for all $i \geq N$. Moreover, $\left\{x_{i}\right\}_{i=N}^{\infty}$ stands in a line. (Hence, the limit point $x$ is also an $r$-cut point. We do not always have $\operatorname{deg}(x)=2$.)

Proof. Suppose that for each $N$ there exists $i \geq N$ such that $\operatorname{deg}\left(x_{i}\right) \geq 3$. We may assume that $\operatorname{deg}\left(x_{i}\right) \geq 3$ for all $i$ and $d\left(x, x_{i}\right)>d\left(x, x_{j}\right)$ for all $i<j$. For each $i$, there exists a connected component $O_{i}$ of $\bar{B}_{r}\left(x_{i}\right) \backslash\left\{x_{i}\right\}$ that does not contain $x$ and $x_{i-1}$. Since $O_{i} \cap S_{r}\left(x_{i}\right)$ is nonempty, we have a contradiction to the local compactness at $x$. Thus it follows that $\operatorname{deg}\left(x_{i}\right)=2$ for all sufficiently large $i$. 
Next, we show that $\left\{x_{i}\right\}_{i=N}^{\infty}$ stands in a line for some sufficiently large $N$. Suppose that $\left\{x_{i}\right\}_{i=N}^{\infty}$ does not stand in a line for any large $N$. By taking a subsequence, we may assume that $O_{i}^{\prime} \cap O_{i+1}^{\prime}=\varnothing$ for $i=1,3,5, \ldots$. Since $O_{i}^{\prime} \cap S_{r}\left(x_{i}\right)$ is nonempty, we have a contradiction to the local compactness at $x$.

Proposition 4.23 does not necessarily hold if $X$ is not locally compact.

Example 4.24. Consider the set $\bigcup_{i=1}^{\infty}\{(2 / i, y) \mid 0 \leq y \leq 2\} \cup\{(x, 0) \mid 0 \leq x \leq 2\} \subset \mathbb{R}^{2}$ with the induced distance. Although the point $(2 / i, 1 / i)$ is a 1 -cut point for every $i \in \mathbb{N}$, the limit point $(0,0)$ is not a local cut point.

\section{The Poincaré inequality}

In this section we prove Theorem 1.3. We begin by recalling the definition of a Poincaré inequality of type $(1, p)$. Let $(X, d)$ be a metric space.

Definition 5.1 (upper gradient [Heinonen and Koskela 1998]). Let $u$ be a function on $X$. A Borel function $g: X \rightarrow[0, \infty]$ is called an upper gradient of $u$ if for all paths $\gamma:[0, l] \rightarrow X$ (proportional to arclength), we have

$$
|u(\gamma(l))-u(\gamma(0))| \leq \int_{0}^{l} g(\gamma(t)) d t .
$$

Every function has an upper gradient $g \equiv \infty$, and hence upper gradients are never unique. For a Lipschitz function $u: X \rightarrow \mathbb{R}$, we define $|\nabla u|: X \rightarrow \mathbb{R}$ by

$$
|\nabla u|(x)=\limsup _{y \rightarrow x} \frac{|u(x)-u(y)|}{d(x, y)}
$$

if $x$ is not isolated, and $|\nabla u|(x)=0$ if $x$ is isolated.

Proposition 5.2. If $u$ is a Lipschitz function, then $|\nabla u|$ is an upper gradient of $u$.

Proof. Let $\gamma:[0, l] \rightarrow X$ be a path. The function $u \circ \gamma$ is Lipschitz and hence is differentiable almost everywhere by Rademacher's theorem. Since $\gamma$ is proportional to arclength,

$$
\left|(u \circ \gamma)^{\prime}(t)\right|=\lim _{s \rightarrow t} \frac{|u(\gamma(t))-u(\gamma(s))|}{|t-s|} \leq \lim _{s \rightarrow t} \frac{|u(\gamma(t))-u(\gamma(s))|}{d(\gamma(t), \gamma(s))} \leq|\nabla u|(\gamma(t))
$$

holds for all differentiable points $t \in[0, l]$. Therefore

$$
|u(\gamma(l))-u(\gamma(0))| \leq \int_{0}^{l}\left|(u \circ \gamma)^{\prime}(t)\right| d t \leq \int_{0}^{l}|\nabla u|(\gamma(t)) d t .
$$

Assume that $(X, d, \mu)$ is a complete, locally compact length space equipped with a Borel measure such that $0<\mu\left(B_{r}(x)\right)<+\infty$ holds for all $x \in X$ and all 
$0<r<+\infty$. For $B \subset X$, we denote

$$
u_{B}=f_{B} u d \mu=\frac{1}{\mu(B)} \int_{B} u d \mu .
$$

Definition 5.3 (Poincaré inequality). Let $1 \leq p<\infty$. We say that $(X, d, \mu)$ satisfies a Poincaré inequality of type $(1, p)$ if, for all $R>0$, there exists a constant $C_{P}=C_{P}(p, R)>0$ depending only on $p$ and $R$ such that

$$
f_{B_{r}(x)}\left|u-u_{B_{r}(x)}\right| d \mu \leq C_{P} r\left(f_{B_{r}(x)} g^{p} d \mu\right)^{1 / p}
$$

holds for all $x \in X$, all $0<r \leq R$, all measurable functions $u$, and all upper gradients $g$ of $u$.

In our setting ( $X$ is a length space), a Poincaré inequality of type $(1, p)$ is derived from a "weak" Poincaré inequality of type $(1, p)$ if we assume that $\mu$ is doubling; see [Hajłasz and Koskela 2000] for details.

Remark 5.4. It follows from Hölder's inequality that every metric measure space satisfying a Poincaré inequality of type $(1, p)$ also satisfies one of type $(1, q)$ for all $q \geq p$.

Keith and Zhong [ $\geq 2007]$ proved the following: Let $p>1$. If $\mu$ is doubling and if $(X, d, \mu)$ satisfies a Poincaré inequality of type $(1, p)$, then there is an $\epsilon>0$ such that $(X, d, \mu)$ satisfies a Poincaré inequality of type $(1, q)$ for all $q>p-\epsilon$.

Proof of Theorem 1.3. The proof is by contradiction; suppose that there is a local cut point $x_{0}$ satisfying (1-3). Fix a sufficiently small $r>0$ such that $B_{r}\left(x_{0}\right) \backslash\left\{x_{0}\right\}$ is disconnected. We choose two connected components $O_{1}, O_{2}$ of $B_{r}\left(x_{0}\right) \backslash\left\{x_{0}\right\}$. For sufficiently large numbers $N \in \mathbb{N}$, we define functions $u_{N}: B_{r}\left(x_{0}\right) \rightarrow \mathbb{R}$ as follows. We set $U_{i}=O_{i} \cap\left(B_{r}\left(x_{0}\right) \backslash B_{\left(N \mu\left(O_{i}\right)\right)^{-1}}\left(x_{0}\right)\right)$ for $i=1,2$ and define

$$
u_{N}(x)= \begin{cases}(-1)^{i+1} / \mu\left(O_{i}\right) & \text { on } U_{i}, \\ (-1)^{i+1} N d\left(x_{0}, x\right) & \text { on } O_{i} \backslash U_{i}, \\ 0 & \text { on } B_{r}\left(x_{0}\right) \backslash\left(O_{1} \cup O_{2}\right) .\end{cases}
$$

The function $u_{N}$ is Lipschitz; hence $\left|\nabla u_{N}\right|$ is an upper gradient of $u_{N}$ (Proposition 5.2). Since $(X, \mu)$ satisfies a Poincaré inequality of type $(1, p)$, for $R \geq r$ there exists a constant $C_{P}=C_{P}(p, R)>0$ such that

$$
f_{B_{r}\left(x_{0}\right)}\left|u_{N}-\left(u_{N}\right)_{B_{r}\left(x_{0}\right)}\right| d \mu \leq C_{P} r\left(f_{B_{r}\left(x_{0}\right)}\left|\nabla u_{N}\right|^{p} d \mu\right)^{1 / p} .
$$


We first estimate the left-hand side of (5-21) from below. We have

$$
\begin{aligned}
& f_{B_{r}\left(x_{0}\right)}\left|u_{N}-\left(u_{N}\right)_{B_{r}\left(x_{0}\right)}\right| d \mu \geq f_{B_{r}\left(x_{0}\right)}\left|u_{N}\right| d \mu-\left|\left(u_{N}\right)_{B_{r}\left(x_{0}\right)}\right| \\
& =\frac{1}{\mu\left(B_{r}\left(x_{0}\right)\right)}\left(\left(\frac{\mu\left(U_{1}\right)}{\mu\left(O_{1}\right)}+\int_{O_{1} \backslash U_{1}} N d\left(x_{0}, x\right) d \mu(x)+\frac{\mu\left(U_{2}\right)}{\mu\left(O_{2}\right)}+\int_{O_{2} \backslash U_{2}} N d\left(x_{0}, x\right) d \mu(x)\right)\right. \\
& \left.-\left|\frac{\mu\left(U_{1}\right)}{\mu\left(O_{1}\right)}+\int_{O_{1} \backslash U_{1}} N d\left(x_{0}, x\right) d \mu(x)-\frac{\mu\left(U_{2}\right)}{\mu\left(O_{2}\right)}-\int_{O_{2} \backslash U_{2}} N d\left(x_{0}, x\right) d \mu(x)\right|\right) \\
& \geq \frac{1}{\mu\left(B_{r}\left(x_{0}\right)\right)}\left(\left(\frac{\mu\left(U_{1}\right)}{\mu\left(O_{1}\right)}+\int_{O_{1} \backslash U_{1}} N d\left(x_{0}, x\right) d \mu(x)+\frac{\mu\left(U_{2}\right)}{\mu\left(O_{2}\right)}+\int_{O_{2} \backslash U_{2}} N d\left(x_{0}, x\right) d \mu(x)\right)\right. \\
& \left.-\left(\left|\frac{\mu\left(U_{1}\right)}{\mu\left(O_{1}\right)}-\frac{\mu\left(U_{2}\right)}{\mu\left(O_{2}\right)}\right|+\int_{O_{1} \backslash U_{1}} N d\left(x_{0}, x\right) d \mu(x)+\int_{O_{2} \backslash U_{2}} N d\left(x_{0}, x\right) d \mu(x)\right)\right) \\
& =\frac{1}{\mu\left(B_{r}\left(x_{0}\right)\right)}\left(\frac{\mu\left(U_{1}\right)}{\mu\left(O_{1}\right)}+\frac{\mu\left(U_{2}\right)}{\mu\left(O_{2}\right)}-\left|\frac{\mu\left(U_{1}\right)}{\mu\left(O_{1}\right)}-\frac{\mu\left(U_{2}\right)}{\mu\left(O_{2}\right)}\right|\right) .
\end{aligned}
$$

Since $N$ is sufficiently large, $\mu\left(U_{i}\right) / \mu\left(O_{i}\right)$ is approximately equal to one $(i=1,2)$. Therefore, we see that the left-hand side of (5-21) is bounded below by the positive number $\mu\left(B_{r}\left(x_{0}\right)\right)^{-1}$, which is independent of $N$.

Next, we bound the right-hand side of (5-21) from above. Note that

$$
\left|\nabla u_{N}\right|= \begin{cases}N & \text { on }\left(O_{1} \backslash U_{1}\right) \cup\left(O_{2} \backslash U_{2}\right), \\ 0 & \text { otherwise }\end{cases}
$$

and $\left(O_{1} \backslash U_{1}\right) \cup\left(O_{2} \backslash U_{2}\right) \subset B_{r_{N}}\left(x_{0}\right)$ for $r_{N}:=\max \left\{\left(N \mu\left(O_{1}\right)\right)^{-1},\left(N \mu\left(O_{2}\right)\right)^{-1}\right\}$. It follows that

$$
\begin{aligned}
C_{P} r\left(f_{B_{r}\left(x_{0}\right)}\left|\nabla u_{N}\right|^{p} d \mu\right)^{1 / p} & =C_{P} r\left(\frac{N^{p}}{\mu\left(B_{r}\left(x_{0}\right)\right)} \mu\left(\left(O_{1} \backslash U_{1}\right) \cup\left(O_{2} \backslash U_{2}\right)\right)\right)^{1 / p} \\
& \leq C_{P} r\left(\frac{N^{p}}{\mu\left(B_{r}\left(x_{0}\right)\right)} \mu\left(B_{r_{N}}\left(x_{0}\right)\right)\right)^{1 / p}
\end{aligned}
$$

which goes to zero as $N \rightarrow \infty$, by assumption (1-3). This is a contradiction.

Let $\alpha>0$. Consider the metric measure space

$$
\left\{\left(x_{1}, x_{2}, \ldots, x_{n}\right) \in \mathbb{R}^{n} \mid x_{1}^{2}+x_{2}^{2}+\cdots+x_{n-1}^{2} \leq x_{n}^{2 \alpha}\right\}
$$

equipped with the Euclidean distance and the $n$-dimensional Lebesgue measure $\mathscr{L}^{n}$. The origin $o=(0,0, \ldots, 0)$ is a local cut point. We have $\mathscr{L}^{n}\left(B_{r}(o)\right)=$ 
$c(n) r^{\alpha(n-1)+1}$. It follows from Theorem 1.3 that, if the metric measure space satisfies a Poincaré inequality of type $(1, p)$, then we have

$$
\alpha \leq \frac{p-1}{n-1} .
$$

\section{Acknowledgments}

The author thanks Professor Takashi Shioya for his constant encouragement and valuable discussions. He thanks Professor Tobias Holck Colding for useful comments. He is indebted to Professor Koichi Nagano for stimulating his interest in the geometry of metric spaces. He also thanks Professor Shin-ichi Ohta and Mr. Shouhei Honda for valuable suggestions related to Theorem 1.3.

\section{References}

[Ambrosio and Tilli 2004] L. Ambrosio and P. Tilli, Topics on analysis in metric spaces, Oxford Lecture Series in Mathematics and its Applications 25, Oxford University Press, Oxford, 2004. MR 2004k:28001 Zbl 1080.28001

[Burago et al. 2001] D. Burago, Y. Burago, and S. Ivanov, A course in metric geometry, Graduate Studies in Mathematics 33, American Mathematical Soc., Providence, RI, 2001. MR 2002e:53053 Zbl 0981.51016

[Buser 1982] P. Buser, "A note on the isoperimetric constant", Ann. Sci. École Norm. Sup. (4) 15:2 (1982), 213-230. MR 84e:58076 Zbl 0501.53030

[Cheeger and Colding 1996] J. Cheeger and T. H. Colding, "Lower bounds on Ricci curvature and the almost rigidity of warped products", Ann. of Math. (2) 144:1 (1996), 189-237. MR 97h:53038 Zbl 0865.53037

[Cheeger and Colding 1997] J. Cheeger and T. H. Colding, "On the structure of spaces with Ricci curvature bounded below. I”, J. Differential Geom. 46:3 (1997), 406-480. MR 98k:53044 Zbl 0902.53034

[Cheeger and Colding 2000a] J. Cheeger and T. H. Colding, "On the structure of spaces with Ricci curvature bounded below. II”, J. Differential Geom. 54:1 (2000), 13-35. MR 2003a:53043 Zbl 1027.53042

[Cheeger and Colding 2000b] J. Cheeger and T. H. Colding, "On the structure of spaces with Ricci curvature bounded below. III”, J. Differential Geom. 54:1 (2000), 37-74. MR 2003a:53044 Zbl 1027.53043

[Cheeger and Gromoll 1971/72] J. Cheeger and D. Gromoll, "The splitting theorem for manifolds of nonnegative Ricci curvature”, J. Differential Geometry 6 (1971/72), 119-128. MR 46 \#2597 Zbl 0223.53033

[Fukaya 1987] K. Fukaya, "Collapsing of Riemannian manifolds and eigenvalues of Laplace operator”, Invent. Math. 87:3 (1987), 517-547. MR 88d:58125 Zbl 0589.58034

[Gromov 1999] M. Gromov, Metric structures for Riemannian and non-Riemannian spaces, Progress in Mathematics 152, Birkhäuser, Boston, 1999. MR 2000d:53065 Zbl 0953.53002

[Hajłasz and Koskela 2000] P. Hajłasz and P. Koskela, "Sobolev met Poincaré", Mem. Amer. Math. Soc. 145:688 (2000), $\mathrm{x}+101$. MR 2000j:46063 Zbl 0954.46022 
[Heinonen and Koskela 1998] J. Heinonen and P. Koskela, "Quasiconformal maps in metric spaces with controlled geometry”, Acta Math. 181:1 (1998), 1-61. MR 99j:30025 Zbl 0915.30018

[Keith and Zhong $\geq 2007$ ] S. Keith and X. Zhong, "The Poincaré inequality is an open ended condition", Ann. of Math.. To appear.

[Kuwae and Shioya 2001] K. Kuwae and T. Shioya, "On generalized measure contraction property and energy functionals over Lipschitz maps", Potential Analysis 15 (2001), 105-121. ICPA98, Hammamet. MR 2002f:31022 Zbl 0996.31006

[Kuwae et al. 2001] K. Kuwae, Y. Machigashira, and T. Shioya, "Sobolev spaces, Laplacian, and heat kernel on Alexandrov spaces", Math. Z. 238:2 (2001), 269-316. MR 2002m:58052 Zbl 1001. 53017

[Lott and Villani 2007] J. Lott and C. Villani, "Weak curvature conditions and functional inequalities”, J. Funct. Anal. 245:1 (2007), 311-333. MR 2311627 Zbl 1119.53028

[Lott and Villani $\geq 2007$ ] J. Lott and C. Villani, "Ricci curvature for metric-measure spaces via optimal transport", Ann. of Math.. To appear.

[Menguy 2000a] X. Menguy, "Examples of nonpolar limit spaces", Amer. J. Math. 122:5 (2000), 927-937. MR 2001i:53058 Zbl 0981.53019

[Menguy 2000b] X. Menguy, "Noncollapsing examples with positive Ricci curvature and infinite topological type”, Geom. Funct. Anal. 10:3 (2000), 600-627. MR 2001g:53074 Zbl 0971.53030

[Menguy 2001] X. Menguy, "Examples of strictly weakly regular points", Geom. Funct. Anal. 11:1 (2001), 124-131. MR 2002c:53054 Zbl 0990.53025

[Ohta 2007] S.-i. Ohta, "On the measure contraction property of metric measure spaces", Comment. Math. Helv. 82:4 (2007), 805-828.

[von Renesse 2007] M.-K. von Renesse, “On local Poincaré via transportation”, in Math. Z. (2007). To appear.

[Saloff-Coste 2002] L. Saloff-Coste, Aspects of Sobolev-type inequalities, London Mathematical Society Lecture Note Series 289, Cambridge University Press, Cambridge, 2002. MR 2003c:46048 Zbl 0991.35002

[Shioya 1999] T. Shioya, "The limit spaces of two-dimensional manifolds with uniformly bounded integral curvature", Trans. American Mathematical Soc. 351:5 (1999), 1765-1801. MR 99h:53052 Zbl 0942.53027

[Sturm 2006a] K.-T. Sturm, "On the geometry of metric measure spaces. I", Acta Math. 196:1 (2006), 65-131. MR 2007k:53051a Zbl 1105.53035

[Sturm 2006b] K.-T. Sturm, "On the geometry of metric measure spaces. II", Acta Math. 196:1 (2006), 133-177. MR 2007k:53051b Zbl 1106.53032

Received September 15, 2006.

\author{
MASAYOSHI WATANABE \\ MATHEMATICAL InSTITUTE \\ TOHOKU UNIVERSITY \\ SENDAI 980-8578 \\ JAPAN \\ sa3m33@math.tohoku.ac.jp
}

